\title{
Energy-Efficient Optimization for HARQ Schemes over Time-Correlated Fading Channels
}

\author{
Zheng Shi, Shaodan Ma, Guanghua Yang, and Mohamed-Slim Alouini
}

\begin{abstract}
Energy efficiency of three common hybrid automatic repeat request (HARQ) schemes including Type I HARQ, HARQ with chase combining (HARQ-CC) and HARQ with incremental redundancy (HARQ-IR), is analyzed and joint power allocation and rate selection to maximize the energy efficiency is investigated in this paper. Unlike prior literature, time-correlated fading channels is considered and two widely concerned quality of service $(\mathrm{QoS})$ constraints, i.e., outage and goodput constraints, are also considered in the optimization, which further differentiates this work from prior ones. Using a unified expression of asymptotic outage probabilities, optimal transmission powers and optimal rate are derived in closed-forms to maximize the energy efficiency while satisfying the QoS constraints. These closed-form solutions then enable a thorough analysis of the maximal energy efficiencies of various HARQ schemes. It is revealed that with low outage constraint, the maximal energy efficiency achieved by Type I HARQ is $\frac{1}{4 \ln 2}$ bits/J, while HARQ-CC and HARQ-IR can achieve the same maximal energy efficiency as $\frac{\kappa_{\infty}}{4 \ln 2} \mathbf{b i t s} / \mathbf{J}$ where $\kappa_{\infty}=1.6617$. Moreover, time correlation in the fading channels has a negative impact on the energy efficiency, while large maximal allowable number of transmissions is favorable for the improvement of energy efficiency. The effectiveness of the energy-efficient optimization is verified by extensive simulations and the results also show that HARQ-CC can achieve the best tradeoff between energy efficiency and spectral efficiency among the three HARQ schemes.
\end{abstract}

Index Terms-Energy efficiency, time-correlated fading channels, hybrid automatic repeat request, power allocation and rate selection.

\section{INTRODUCTION}

$\mathbf{T}$ HE past few years have witnessed an explosive growth in wireless data traffic and the number of mobile terminals. We are therefore obliged to continuously enhance the spectral/energy efficiency of wireless communication systems to meet the ever-increasing demand. Towards this end, adaptive

Copyright (c) 2015 IEEE. Personal use of this material is permitted. However, permission to use this material for any other purposes must be obtained from the IEEE by sending a request to pubs-permissions@ieee.org

This work was supported in part by National Natural Science Foundation of China under grant 61601524, in part by the Macau Science and Technology Development Fund under grants 091/2015/A3 and 020/2015/AMJ, and in part by the Research Committee of University of Macau under grants MYRG201400146-FST and MYRG2016-00146-FST. (Corresponding author: Guanghua Yang.)

Zheng Shi is with the School of Electrical and Information Engineering and the Institute of Physical Internet, Jinan University (Zhuhai campus), Zhuhai 519070, China (e-mail:shizheng0124@gmail.com).

Shaodan Ma is with the Department of Electrical and Computer Engineering, University of Macau, Macao (e-mail: shaodanma@umac.mo).

Guanghua Yang is with Institute of Physical Internet, Jinan University (Zhuhai Campus), Zhuhai, China (e-mail: ghyang@jnu.edu.cn).

Mohamed-Slim Alouini is with the Computer, Electrical, and Mathematical Science and Engineering (CEMSE) Division, King Abdullah University of Science and Technology (KAUST) Thuwal, Makkah Province, Saudi Arabia (e-mail:slim.alouini@kaust.edu.sa). modulation and coding (AMC) and hybrid automatic repeat request (HARQ) are recognized as two promising techniques so far [1], [2]. In particular, AMC is an efficient physicallayer technique that adapts the modulation type, the transmission rate and even the transmission power to channel state information (CSI) available at the transmitter [3]. However, with only statistical or limited CSI at the transmitter, HARQ turns out to be more efficient to offer higher throughput and/or lower outage probability with the aid of multiple transmissions adaptive to channel conditions [4]. Specifically, HARQ enables reliable and robust data transmissions through leveraging forward error correction at the physical layer as well as automatic repeat request at the link layer. Generally, HARQ techniques are classified into three types based on the encoding and decoding operations at the transceivers, i.e., Type I HARQ, HARQ with chase combining (HARQ-CC) and HARQ with incremental redundancy (HARQ-IR) [5]. The key difference among them is that Type I HARQ discards the erroneously received sub-codewords and performs memoryless decoding at the receiver, whereas HARQ-CC and HARQ-IR utilize the erroneous sub-codewords for subsequent decoding through chase combining and code combining, respectively. They provide various tradeoffs between performance and complexity and have a wide range of applications in wireless communications.

To further boost the performance of HARQ schemes when statistical/limited CSI is available, optimal design of HARQ system has sparked of considerable research interests lately. The majority of the optimal designs in the literature aim to maximize the spectral efficiency which is commonly quantified by long-term average throughput (LTAT) [6]-[14]. For example, cooperative HARQ scheme is investigated and optimal rate and transmission powers are found to maximize the LTAT through numerical search in [6]. Noticing no closedform solution and heavy computational overhead in [6], two suboptimal algorithms are then proposed for rate selection in [7] to substantially reduce the computational complexity while guaranteeing a comparable performance to the globally optimal solution. By deriving the throughput using Laplace transform, a parameterization-based method is developed to find the optimal rate in (semi-)closed-form to maximize the throughput for various HARQ schemes in [10]. Moreover, when outdated CSI is available at the transmitter, the optimal transmission rate to maximize the LTAT of HARQ-IR is found by using dynamic programming in [12]. Numerical results demonstrate that a notable throughput gain can be achieved by this optimal design.

Apart from spectral efficiency, there is another performance 
metric which becomes of increasing concern in wireless communications, that is, energy efficiency [14]-[23]. It is particularly important in energy limited networks, e.g., internet-ofthings (IoT) networks. However, there are very few optimal designs aiming at energy efficiency maximization in the literature. Specifically, in [16], three optimum schemes are proposed to achieve various tradeoffs between the energy efficiency and the spectral efficiency for Type I HARQ under quasistatic fading channels. A fixed power transmission scheme is proposed to maximize the energy efficiency of HARQ-IR under independent fading channels and the optimal power is found numerically in [14]. On the other hand, it is revealed in [17], [18] that the maximal achievable energy efficiency of Type I HARQ is $\frac{1}{\mathrm{e} \ln 2} \mathrm{bits} / \mathrm{J}$, while HARQ-CC and HARQ-IR can achieve the same maximal energy efficiency as $\frac{1}{\ln 2} \mathrm{bits} / \mathrm{J}$. Unfortunately, these prior analyses and optimal designs rarely consider quality of service (QoS) constraints (e.g., outage probability and throughput constraints) while maximizing the energy efficiency. Such QoS constraints are practical and usually should be satisfied for practical applications. They would definitely deteriorate the energy efficiency due to the shrinking of the feasible region in the optimization. Moreover, all the energy-efficient designs are applicable to either quasistatic fading channels or independent fading channels, but not optimal to time-correlated fading channels which usually occur in low-to-medium mobility environments [24], [25]. Timecorrelation among fading channels usually causes negative impacts on the system performance, and it has been intensively studied for various systems in the literature. Specifically, the performance of HARQ-IR over time-correlated Rayleigh fading channels is investigated in [26] through polynomial fitting technique. The analytical results are further extended to cooperative HARQ-IR over time-correlated Nakagami-m fading channels in [27]. Most of prior works are conducted for the analysis of either outage probability or spectral efficiency. To our best knowledge, analysis of energy-efficiency and its optimization for HARQ schemes over time-correlated fading channels has not been discussed and solved yet.

Here we thus take a step further to investigate energyefficient optimization for various HARQ schemes over timecorrelated fading channels with QoS constraints, particularly, a tolerable outage constraint and a minimum goodput constraint. A joint power allocation and rate selection scheme is develope$\mathrm{d}$ to maximize the energy efficiency while satisfying the QoS constraints. With a unified expression of asymptotic outage probabilities, the optimal transmission powers and rates for the three common HARQ schemes including Type I HARQ, HARQ-CC and HARQ-IR are derived in closed-forms. The closed-form optimal solutions then enable a thorough analysis of the maximum energy efficiency when time-correlated fading channels and QoS constraints are considered. It is found that the channel time correlation has a negative impact on the energy efficiency, while large maximal allowable number of transmissions is beneficial to the energy efficiency. More importantly, it is revealed that with low outage constraint, the maximal energy efficiency of Type I HARQ is $\frac{1}{4 \ln 2} \mathrm{bits} / \mathrm{J}$, while both HARQ-CC and HARQ-IR can achieve a maximal energy efficiency of $\frac{\kappa_{\infty}}{4 \ln 2}$ bits/J where $\kappa_{\infty}=1.6617$. Noticing that the maximization of the energy efficiency and the spectral efficiency are generally two conflicting goals, the tradeoff between the spectral efficiency and the energy efficiency is also discussed. Numerical results finally demonstrate the effectiveness of our energy-efficient optimization and show that HARQ-CC in fact can achieve a better tradeoff between the energy efficiency and the spectral efficiency than Type I HARQ and HARQ-IR.

The rest of this paper is organized as follows. Section II introduces the system model. An energy-efficient optimization is then proposed for the three HARQ schemes and the optimal transmission powers are found in closed-form in Section III. In Section IV, the optimal rates are derived in closed-form for three HARQ schemes and their corresponding optimal energy efficiencies are thoroughly analyzed. Section V presents the numerical results for verification and investigates the tradeoff between the energy efficiency and the spectral efficiency of our design. Finally, Section VI concludes this paper.

\section{System MODEL}

This paper considers a point-to-point wireless communication system with HARQ transmissions. Following HARQ protocol, the source first encodes $N_{b}$ information bits into a mother codeword of length $L N_{s}$, where $L$ denotes the maximal allowable number of transmissions. The codeword is then punctured into $L$ sub-codewords, each with the same length $N_{s}$. These sub-codewords are sequentially transmitted to the destination through multiple rounds. Notice that in Type I HARQ and HARQ-CC, the same sub-codeword is transmitted in each HARQ round, while in HARQ-IR a different subcodeword with new parity bits is transmitted in each HARQ round [8]. For all the three HARQ schemes, the received signal $\mathbf{y}_{l}$ in the $l$ th HARQ round at the destination can be unified as

$$
\mathbf{y}_{l}=\sqrt{P_{l}} h_{l} \mathbf{x}_{l}+\mathbf{z}_{l}, \quad l=1,2, \cdots, L,
$$

where $\mathbf{x}_{l}$ denotes a sub-codeword with length of $N_{s}$ and each symbol of $\mathbf{x}_{l}$ follows Gaussian distribution with unit average energy, i.e., $\mathrm{E}\left\{\mathbf{x}_{l}{ }^{\mathrm{T}} \mathbf{x}_{l}\right\}=N_{s} ; P_{l}$ stands for the transmission power in the $l$ th HARQ round; $\mathbf{z}_{l}$ denotes complex Gaussian white noise vector whose elements have zero mean and unit variance; and $h_{l}$ represents Rayleigh fading channel coefficient in the $l$ th HARQ round. Under low-to-medium mobility environments, wireless communications usually experiences timecorrelated block-fading channels. To accommodate this channel time correlation, a widely used time-correlated Rayleigh fading channel model given in [24] is adopted here

$$
h_{l}=\sigma_{l}\left(\sqrt{1-\rho^{2(l-1)}} \mathfrak{h}_{l}+\rho^{l-1} \mathfrak{h}_{0}\right), \quad l=1,2, \cdots, L,
$$

where $\rho$ and $\sigma_{l}^{2}$ denote time correlation coefficient and the variance of $h_{l}$, respectively; and $\mathfrak{h}_{0}, \mathfrak{h}_{1}, \cdots, \mathfrak{h}_{L}$ follow independent circularly-symmetric complex Gaussian distribution with zero mean and unit variance, i.e., $\mathfrak{h}_{0}, \mathfrak{h}_{l} \sim \mathcal{C N}(0,1)$. This model is general and covers quasi-static fading channels (i.e., fully correlated fading channels where $h_{1}=h_{2}=\cdots=h_{L}$ ) and fast fading channels (i.e., independent fading channels where $h_{1}, h_{2}, \cdots, h_{L}$ are independent) as special cases with $\rho=1$ and $\rho=0$, respectively. In particular, it is worth 
highlighting that the fixed-rate HARQ-IR is considered in this paper for the sake of simplicity and fair comparison, that is, the length of each sub-codeword delivered in HARQ-IR scheme keeps fixed throughout HARQ rounds. Hereby, as the length of each sub-codeword is $N_{s}$ for all the HARQ schemes, the initial transmission rate that denotes the data transmission rate in the first HARQ round is $R=\frac{N_{b}}{N_{s}}$.

It is assumed that perfect instantaneous CSI is available at the receiver, but only the statistical CSI is available at the transmitter. To boost the communication performance, the transmission powers $P_{1}, \cdots, P_{L}$ and the transmission rate $R$ should be properly designed by using the statistical CSI at the transmitter. In this paper, energy efficiency is concerned and our design objective is to maximize the energy efficiency under QoS constraints by jointly optimizing transmission powers and rate. Since the energy efficiency and QoS constraints are generally defined based on the most fundamental performance metric, i.e., outage probability, it is discussed first in the following. For HARQ schemes, an outage event happens when the destination fails to decode the message after $L$ transmissions. Here three common HARQ schemes are particularly discussed: Type I HARQ, HARQ-CC and HARQ-IR. They are differentiated by their encoding and decoding operations at the transceiver. Specifically, Type I HARQ only takes the received signal in the latest round for decoding, HARQ-CC adopts maximum ratio combining of the received signals in multiple rounds for joint decoding, while HARQ-IR adopts code combining of the received signals in multiple rounds for joint decoding. Therefore the outage probabilities of the three HARQ schemes are different and given respectively as (3) at the top of this page [5]. The outage probabilities of the three HARQ schemes under time-correlated fading channels have been derived in [28]-[30]. However, their expressions are too complex and involve complicated special functions, which provide little insights and hinder the optimal design based on them. Fortunately, by assuming Gaussian codes and typical set decoding [5], the asymptotic outage probabilities of Type I HARQ and HARQ-CC under time-correlated Rayleigh fading channels have been exactly derived in simple forms in [28], [29], respectively, while the asymptotic outage probability of HARQ-IR has been exactly derived in closed-form in [30]. It has been shown that the asymptotic outage probabilities match well with the exact outage probabilities under low outage region or high SNR. They can be unified as

$$
p_{\text {out }, L} \simeq \phi_{L}\left(\prod_{k=1}^{L} P_{k}\right)^{-1}
$$

where $p_{\text {out }, 0}=1$ if $R>0$ and $p_{\text {out }, 0}=0$ otherwise, " $\simeq$ " stands for the asymptotically equal operator, and $\phi_{L}$ changes among various HARQ schemes and is explicitly given by

$$
\phi_{L}= \begin{cases}\varsigma_{L}\left(2^{R}-1\right)^{L}, & \text { Type I - HARQ } \\ \frac{\varsigma_{L}\left(2^{R}-1\right)^{L}}{\Gamma(L+1)}, & \text { HARQ - CC } \\ \varsigma_{L} g_{L}(R), & \text { HARQ - IR },\end{cases}
$$

where $\phi_{0}=1$ if $R>0$ and $\phi_{0}=0$ otherwise. In (5), $\Gamma(\cdot)$ denotes Gamma function, $\varsigma_{L}$ quantifies the impact of time-correlated fading channels, i.e., $\varsigma_{L}=\frac{(\ell(L, \rho))^{-1}}{\prod_{k=1}^{L} \sigma_{k}{ }^{2}}$ in which $\ell(L, \rho)$ particularly quantifies the impact of time correlation with

$$
\ell(L, \rho)=\left(1+\sum_{k=1}^{L} \frac{\rho^{2(k-1)}}{1-\rho^{2(k-1)}}\right) \prod_{k=1}^{L}\left(1-\rho^{2(k-1)}\right),
$$

and $\ell(0, \rho)=1$. Hence $\varsigma_{0}=1$. Moreover, in (5), if $R=0$ and $L=0,\left(2^{R}-1\right)^{L}=0$ by convention, and $g_{L}(R)$ is given by

$$
\begin{aligned}
g_{L}(R) & =\frac{1}{2 \pi \mathrm{i}} \int_{a-\mathrm{i} \infty}^{a+\mathrm{i} \infty} \frac{2^{R s}}{s(s-1)^{L}} d s \\
& =(-1)^{L}+2^{R} \sum_{k=0}^{L-1}(-1)^{k} \frac{(R \ln 2)^{L-k-1}}{(L-k-1) !}
\end{aligned}
$$

with $\mathrm{i}=\sqrt{-1}, a>1$ and $g_{0}(0)=0$. The unified asymptotic outage probability in (4) not only provides clear insights but also enables optimal design of the transmission powers and transmission rate analytically to achieve various objectives. It will lead to closed-form optimal solutions and is adopted here for our optimal design. From the simulation results shown later, the optimal design based on the asymptotic outage probabilities can achieve similar performance to that based on the exact outage probabilities through numerical search, but with much lower complexity.

\section{ENERGY-EFFICIENT OPTIMIZATION}

As defined in [14], [15], energy efficiency quantifies the amount of successfully delivered information per unit energy. Specifically, for HARQ schemes with maximum $L$ transmissions, based on the renewal-reward theorem [31], the energy efficiency $\eta_{L}$ can be written as the ratio of the average number of correctly received bits $\bar{N}_{b}=N_{b}\left(1-p_{\text {out }, L}\right)$ to the average amount of energy used $\overline{\mathcal{E}}=\sum_{l=1}^{L} p_{\text {out }, l-1} P_{l} \mathrm{E}\left\{\mathbf{x}_{l}{ }^{\mathrm{T}} \mathbf{x}_{l}\right\}=$ $N_{s} \sum_{l=1}^{L} p_{\text {out }, l-1} P_{l}$, i.e.,

$$
\eta_{L}=\frac{\bar{N}_{b}}{\overline{\mathcal{E}}}=\frac{N_{b}\left(1-p_{\text {out }, L}\right)}{N_{s} \bar{P}}=\frac{\mathcal{T}_{g}}{\bar{P}},
$$

where $\bar{P}=\sum_{l=1}^{L} p_{\text {out }, l-1} P_{l}$ is the average total transmission power and $\mathcal{T}_{g}=R\left(1-p_{\text {out }, L}\right)$. It is worth noting that $\mathcal{T}_{g}$ is frequently termed as the goodput/effecitve rate, which is an important performance metric to evaluate the throughput of HARQ schemes [32], [33]. As pointed out in [32], the goodput denotes the number of bits successfully delivered per packet transmission. It is asymptotically equivalent to the spectral efficiency under high SNR. Notice that the spectral efficiency denotes the average number of successfully delivered bits per channel use and its definition will be given later.

With statistical CSI at the transmitter, the transmission powers $P_{1}, \cdots, P_{L}$ and the transmission rate $R$ could be optimally designed to maximize the energy efficiency $\eta_{L}$. In the literature, some optimal/sub-optimal designs have been proposed for HARQ schemes [14], [16]. Unfortunately, most of them are applicable for independent fading channels and rarely consider QoS constraints. Considering the wide occurrences of time-correlated fading channels and QoS requirements in practice, we take a step forward to incorporate both of them in 


$$
p_{\text {out }, L}= \begin{cases}\operatorname{Pr}\left(\log _{2}\left(1+\max \left(P_{1}\left|h_{1}\right|^{2}, P_{2}\left|h_{2}\right|^{2}, \cdots, P_{L}\left|h_{L}\right|^{2}\right)\right) \leq R\right), & \text { Type I- HARQ } \\ \operatorname{Pr}\left(\log _{2}\left(1+\sum_{l=1}^{L} P_{l}\left|h_{l}\right|^{2}\right) \leq R\right), & \text { HARQ - CC } \\ \operatorname{Pr}\left(\sum_{l=1}^{L} \log _{2}\left(1+P_{l}\left|h_{l}\right|^{2}\right) \leq R\right), & \text { HARQ - IR. }\end{cases}
$$

the energy-efficient optimization. Two widely concerned QoS constraints are particularly considered here. One is the outage constraint, i.e., $p_{\text {out }, L} \leq \varepsilon$, and the other is minimum goodput constraint ${ }^{1}$, i.e., $\mathcal{T}_{g} \geq \mathcal{T}_{0}$. With these QoS constraints, the optimum design of transmission powers and transmission rate to maximize the energy efficiency can be formulated as

$$
\begin{array}{cl}
\max _{P_{1}, \cdots P_{L}, R} & \eta_{L} \\
\text { subject to } & p_{\text {out }, L} \leq \varepsilon \\
& \mathcal{T}_{g} \geq \mathcal{T}_{0} \\
& P_{l} \geq 0, \quad 1 \leq l \leq L \\
& R \geq 0,
\end{array}
$$

where $\varepsilon$ and $\mathcal{T}_{0}$ denote the maximal allowable outage probability and the minimum required goodput, respectively. It is clear that (9) is a fractional programming problem. Due to the complicated expressions of the energy efficiency and outage probability under time-correlated fading channels, the optimal design is very challenging and it is difficult to find the optimal solution directly. But by introducing an auxiliary variable $\alpha=p_{\text {out }, L}$ (target outage probability), the fractional programming problem can be reformulated as

$$
\begin{array}{ll}
\max _{P_{1}, \cdots P_{L}, R, \alpha} & \frac{R(1-\alpha)}{\bar{P}} \\
\text { subject to } & p_{\text {out }, L}=\alpha \\
& 0 \leq \alpha \leq \varepsilon \\
& R(1-\alpha) \geq \mathcal{T}_{0} \\
& P_{l} \geq 0, \quad 1 \leq l \leq L \\
& R \geq 0,
\end{array}
$$

which can be further decomposed into three subproblems equivalently with regard to transmission powers $P_{1}, \cdots, P_{L}$, target outage probability $\alpha$ and transmission rate $R$, respectively [34, Eqs.11-13]. It should be noted that this equivalent decomposition holds without the necessity of any conditions. They can be solved individually in closed-forms in the following.

\section{A. Optimal Power Allocation}

Given the transmission rate $R$ and the target outage probability $\alpha$, the problem in (10) reduces to the design of the transmission powers as

$$
\begin{array}{ll}
\min _{P_{1}, \cdots P_{L}} & \bar{P} \\
\text { subject to } & p_{\text {out }, L}=\alpha \\
& P_{l} \geq 0,1 \leq l \leq L .
\end{array}
$$

\footnotetext{
${ }^{1}$ The goodput constraint is asymptotically equivalent to the spectral efficiency constraint [32]. Since the goodput expression is simpler than the spectral efficiency, the consideration of goodput constraint will simplify the optimization and lead to close-form solution without loss of the practical significance.
}

This optimization problem is similar to the power allocation problem in [29], except that the equality outage constraint $p_{\text {out }, L}=\alpha$ is changed as inequality outage constraint $p_{\text {out }, L} \leq \alpha$. It has been proved in [29] that the minimal average total transmission power with inequality outage constraint is achieved at the outage boundary, i.e., $p_{\text {out }, L}=\alpha$. Therefore, the closed-form optimal power solution in [29] is applicable to our power design problem in (11). Specifically, as shown in [29], the optimal powers are given as functions of the transmission rate $R$ and target outage probability $\alpha$ as

$$
\begin{gathered}
P_{L}^{*}=\left(\frac{\phi_{L} \prod_{k=2}^{L}\left(\frac{2 \phi_{k-1}}{\phi_{k-2}}\right)^{2^{1-k}}}{2^{L-1} \alpha \phi_{L-1}}\right)^{\frac{2^{L-1}}{2^{L}-1}}, \\
P_{l}^{*}=\prod_{k=l+1}^{L}\left(\frac{2 \phi_{k-1}}{\phi_{k-2}}\right)^{2^{l-k}} P_{L}^{* 2^{l-L}}, \quad 1 \leq l \leq L-1,
\end{gathered}
$$

and the minimal average total transmission power $\bar{P}^{*}$ can be obtained in a simple form as

$$
\bar{P}^{*}=\frac{\left(2^{L}-1\right) \alpha^{-\frac{1}{2^{L}-1}}}{2^{\frac{L}{1-2^{-L}}-2}}\left(\prod_{k=1}^{L}\left(\frac{\phi_{k}}{\phi_{k-1}}\right)^{2^{-k}}\right)^{\frac{1}{1-2^{-L}}} .
$$

Clearly from (12), (13) and (14), the decrease of the target outage probability $\alpha$ will lead to the increase of transmission powers $P_{l}^{*}$ and then the increase of the minimal average total transmission power $\bar{P}^{*}$. In addition, it can be found that the minimal average total transmission power $\bar{P}^{*}$ becomes irrelevant to the target outage probability $\alpha$ when $L \rightarrow \infty$.

\section{B. Optimal Outage Probability}

Now putting the optimal powers (12) and (13) into the original optimization problem (10), the original problem can be reduced to the optimization of two variables of the transmission rate $R$ and target outage probability $\alpha$ as

$$
\begin{array}{cl}
\max _{R, \alpha} & \frac{R(1-\alpha)}{\bar{P}^{*}} \\
\text { subject to } & 0 \leq \alpha \leq \varepsilon \\
& R(1-\alpha) \geq \mathcal{T}_{0} \\
& R \geq 0 .
\end{array}
$$

With (14), the objective function of (15) can be rewritten as

$\frac{R(1-\alpha)}{\bar{P}^{*}}=\underbrace{\frac{2^{\frac{L}{1-2^{-L}}-2}}{\left(2^{L}-1\right)}}_{\triangleq \psi} \underbrace{(1-\alpha) \alpha^{\frac{1}{L^{L}-1}}}_{\triangleq f(\alpha)} \frac{R}{\left(\prod_{k=1}^{L}\left(\frac{\phi_{k}}{\phi_{k-1}}\right)^{2^{-k}}\right)^{\frac{1}{1-2^{-L}}}}$.

It is clear that the target outage probability is only involved in the term $f(\alpha)$ in (16). When given the transmission rate 
$R$, the problem in (15) is equivalent to the following target outage probability optimization as

$$
\begin{array}{ll}
\max _{\alpha} & f(\alpha) \\
\text { subject to } & 0 \leq \alpha \leq \varepsilon \\
& R(1-\alpha) \geq \mathcal{T}_{0},
\end{array}
$$

whose feasibility and optimal solution can be found in the following theorem.

Theorem 1. The optimization problem (17) is infeasible when $R \leq \mathcal{T}_{0}$. When $R>\mathcal{T}_{0}$, the optimal target outage probability is $\alpha^{*}=\min \left\{\varepsilon, 1-\frac{\mathcal{T}_{0}}{R}, 2^{-L}\right\}$ and the optimal $f\left(\alpha^{*}\right)$ can be written as

$$
\begin{aligned}
f\left(\alpha^{*}\right)= & \frac{\mathcal{T}_{0}}{R}\left(1-\frac{\mathcal{T}_{0}}{R}\right)^{c}\left(\chi\left(R-\mathcal{T}_{0}\right)-\chi\left(R-\frac{\mathcal{T}_{0}}{1-\Delta}\right)\right) \\
& +(1-\Delta) \Delta^{c} \chi\left(R-\frac{\mathcal{T}_{0}}{1-\Delta}\right),
\end{aligned}
$$

where $c=\frac{1}{2^{L}-1}, \Delta=\min \left\{\varepsilon, 2^{-L}\right\}$, and $\chi(t)$ is an indicator function as

$$
\chi(t)=\left\{\begin{array}{ll}
0 & t<0 \\
1 & t \geq 0
\end{array} .\right.
$$

Proof: Please see Appendix A.

\section{Optimal Rate Selection}

After determining the optimal target outage probability $\alpha^{*}$ and considering the feasible region of $R>\mathcal{T}_{0}$ in Theorem 1, the energy-efficient optimization in (15) is finally reduced to the optimal rate selection as

$$
\max _{R} \quad \eta_{L}=\psi f\left(\alpha^{*}\right) \frac{R}{\left(\prod_{k=1}^{L}\left(\frac{\phi_{k}}{\phi_{k-1}}\right)^{2^{-k}}\right)^{\frac{1}{1-2^{-L}}}}
$$$$
\text { subject to } R>\mathcal{T}_{0} \text {. }
$$

Plugging (18) into (20), although the optimal transmission rate $R^{*}$ can be computed numerically through one dimensional search, it is lacking of clear insights. In this paper, we aim to derive the optimal rate in closed-form and extract clear insights for energy-efficient optimization. Since $\phi_{k}$ in the objective function depends on the transmission rate $R$ as shown in (5) and changes among different HARQ schemes, the optimal rate selection for various HARQ schemes should be investigated individually and will be discussed one by one in the next section.

\section{Optimal Rate AND Optimal Energy EFficiency}

\section{A. Type I $H A R Q$}

1) Optimal Rate: Putting (5) into the objective function in (20), the energy efficiency of Type I HARQ can be written as

$$
\eta_{I, L}=\psi \theta_{L} \frac{f\left(\alpha^{*}\right) R}{2^{R}-1}
$$

where

$$
\begin{aligned}
\theta_{L} & =\left(\prod_{k=1}^{L}\left(\frac{\varsigma_{k-1}}{\varsigma_{k}}\right)^{2^{-k}}\right)^{\frac{1}{1-2^{-L}}} \\
& =\left(\prod_{k=1}^{L}\left(\frac{\ell(k, \rho) \sigma_{k}^{2}}{\ell(k-1, \rho)}\right)^{2^{-k}}\right)^{\frac{1}{1-2^{-L}}}
\end{aligned}
$$

Substituting (18) into (21) yields

$$
\begin{aligned}
\eta_{I, L}= & \psi \theta_{L} \mathcal{T}_{0} \frac{\left(1-\frac{\mathcal{T}_{0}}{R}\right)^{c}}{2^{R}-1}\left(\chi\left(R-\mathcal{T}_{0}\right)-\chi\left(R-\frac{\mathcal{T}_{0}}{1-\Delta}\right)\right) \\
& +\psi \theta_{L}(1-\Delta) \Delta^{c} \frac{R}{2^{R}-1} \chi\left(R-\frac{\mathcal{T}_{0}}{1-\Delta}\right) .
\end{aligned}
$$

From (23), we can see that when $R>\frac{\mathcal{T}_{0}}{1-\Delta}$, the first term is zero and the energy efficiency reduces to $\eta_{I, L}=$ $\psi \theta_{L}(1-\Delta) \Delta^{c} \frac{R}{2^{R}-1}$ which is a decreasing function of $R$. Moreover, the energy efficiency $\eta_{I, L}$ is continuous in the domain of $R>\mathcal{T}_{0}$. Therefore, considering the constraint of $R>\mathcal{T}_{0}$ in (20), we can conclude that the optimal rate $R^{*}$ to achieve the maximal energy efficiency should lie within the range of $\left(\mathcal{T}_{0}, \frac{\mathcal{T}_{0}}{1-\Delta}\right]$. In this range, the second term in (23) is zero and the energy efficiency can be simplified as

$$
\eta_{I, L}=\psi \theta_{L} \mathcal{T}_{0} \frac{\left(1-\frac{\mathcal{T}_{0}}{R}\right)^{c}}{2^{R}-1}
$$

Then the problem of rate selection in (20) is equivalent to a minimization problem as

$$
\begin{array}{ll}
\min _{R} & \Phi(R)=\left(2^{R}-1\right)\left(1-\frac{\mathcal{T}_{0}}{R}\right)^{-c} \\
\text { subject to } & \mathcal{T}_{0}<R \leq \frac{\mathcal{T}_{0}}{1-\Delta} .
\end{array}
$$

The optimal solution to (25) can be found in closed-form and is shown in the following theorem.

Theorem 2. The optimal rate to maximize the energy efficiency while guaranteeing the outage and goodput performance for Type I HARQ is

$$
R^{*}=\min \left\{\frac{\mathcal{T}_{0}}{1-\Delta}, \varphi^{-1}(0)\right\},
$$

where $\varphi^{-1}$ denotes the inverse function with respect to $\varphi(R)=\ln (2) R\left(R-\mathcal{T}_{0}\right) 2^{R}-c \mathcal{T}_{0}\left(2^{R}-1\right)$ and $\varphi^{-1}(0)$ refers to the zero point of $\varphi(R)$. When $\varepsilon \geq 2^{-L}$, the optimal transmission rate reduces to $R^{*}=\varphi^{-1}(0)$.

Proof: Please see Appendix B.

It is noteworthy that the zero point $\varphi^{-1}(0)$ can be easily computed since $\varphi(R)$ is an increasing function of $R$ within the range of $\left(\mathcal{T}_{0}, \infty\right)$.

2) Optimal Energy Efficiency: Putting the optimal rate (26) into (24), the optimal energy efficiency of Type I HARQ can be obtained as

$$
\eta_{I, L}^{*}=\psi \theta_{L} \mathcal{T}_{0} \frac{\left(1-\frac{\mathcal{T}_{0}}{R^{*}}\right)^{c}}{2^{R^{*}}-1}=\psi \theta_{L} \mathcal{T}_{0} \frac{\left(R^{*}-\mathcal{T}_{0}\right)^{c}}{R^{* c}\left(2^{R^{*}}-1\right)} .
$$

As aforementioned, the maximal energy efficiency of HARQ schemes over independent Rayleigh fading channels without QoS constraints has been studied in [18]. It has been proved that the maximal energy efficiency of Type I HARQ operating over independent Rayleigh fading channels without QoS constraints is $\frac{1}{\mathrm{e} \ln (2)}$. From (27), we can see that channel time correlation will affect the optimal energy efficiency through the term $\theta_{L}$. As proved in Appendix C, $\theta_{L}$ is a decreasing function of time correlation coefficient $\rho$. In other words, channel time correlation has a negative impact on the optimal energy efficiency and time-correlated fading channels 
provide lower energy efficiency than time independent fading channels with $\rho=0$. We thus can expect that the maximal energy efficiency of Type I HARQ over time-correlated fading channels with QoS constraints will be lower than $\frac{1}{\mathrm{e} \ln (2)}$ and will be investigated here.

To proceed with the investigation, we first analyze the monotonic property of the optimal energy efficiency with respect to the maximal number of transmissions $L$. From the original energy efficiency maximization problem in (9), we can find the following property.

Property 1. The optimal energy efficiencies of all the three HARQ schemes are non-decreasing functions of the maximal number of transmissions $L$ and $\eta_{L}^{*} \leq \lim _{L \rightarrow \infty} \eta_{L}^{*} \triangleq \eta_{\infty}^{*}$.

Proof: Please see Appendix D.

Notice that this property is applicable to all the three HARQ schemes. For Type I HARQ scheme, the maximal energy efficiency is thus achieved when $L \rightarrow \infty$ and is denoted as $\eta_{I, \infty}^{*}$ which can be found as shown in the following theorem.

Theorem 3. Under time-correlated Rayleigh fading channels, the optimal energy efficiency of Type I HARQ with outage and goodput constraints is upper bounded by

$$
\eta_{I, L}^{*} \leq \eta_{I, \infty}^{*}=\frac{\theta_{\infty} \mathcal{T}_{0}}{4\left(2^{\mathcal{T}_{0}}-1\right)},
$$

where $\theta_{\infty} \triangleq \lim _{L \rightarrow \infty} \theta_{L}$ exists. The maximal energy efficiency $\eta_{I, \infty}^{*}$ is a decreasing function of the goodput threshold $\mathcal{T}_{0}$. Particularly, for Rayleigh fading channels with unit channel gains as $\sigma_{1}{ }^{2}=\cdots=\sigma_{L}{ }^{2}=1, \theta_{\infty} \leq 1$ and the maximal energy efficiency of Type I HARQ with outage and goodput constraints satisfies

$$
\eta_{I, \infty}^{*}=\frac{\theta_{\infty} \mathcal{T}_{0}}{4\left(2^{\mathcal{T}_{0}}-1\right)} \leq \lim _{\mathcal{T}_{0} \rightarrow 0} \frac{\mathcal{T}_{0}}{4\left(2^{\mathcal{T}_{0}}-1\right)}=\frac{1}{4 \ln (2)} \triangleq \eta_{I, \infty}^{\max }
$$

Proof: Please see Appendix E.

It means that when QoS constraints are considered, the maximal energy efficiency which can be achieved by Type I HARQ is $\frac{1}{4 \ln (2)}$.

\section{B. $H A R Q-C C$}

1) Optimal Rate: Similarly to Section IV-A, with the optimal powers $P_{1}^{*}, \cdots, P_{L}^{*}$, the optimal target outage probability $\alpha^{*}$ and the definition in (5), the energy efficiency of HARQ$\mathrm{CC}$ in (20) can be rewritten as

$$
\eta_{C C, L}=\kappa_{L} \frac{1}{1-2^{-L}} \psi \theta_{L} \frac{f\left(\alpha^{*}\right) R}{2^{R}-1},
$$

where $\kappa_{L}=\prod_{k=1}^{L} k^{2^{-k}}$. Noticing that the only difference between (21) and (30) is at an additional term $\kappa_{L}^{\frac{1}{1-2^{-L}}}$ involved in (30) and this term is irrelevant to the transmission rate, the optimal transmission rate $R^{*}$ of HARQ-CC can thus be derived as the same as that for Type I HARQ shown in (26) in Theorem 2.
2) Optimal Energy Efficiency: Accordingly, the optimal energy efficiency of HARQ-CC can be written as

$$
\eta_{C C, L}^{*}=\kappa_{L}^{\frac{1}{1-2^{-L}}} \eta_{I, L}^{*} .
$$

Since $\kappa_{L}>1$, it thus reveals that HARQ-CC surpasses Type I HARQ in terms of the optimal energy efficiency, i.e., $\eta_{C C, L}^{*}>$ $\eta_{I, L}^{*}$.

Moreover, based on Property 1 and Theorem 3, it is easy to get the following result of maximal energy efficiency of HARQ-CC.

Theorem 4. Under time-correlated Rayleigh fading channels, the optimal energy efficiency of HARQ-CC with outage and goodput constraints is upper bounded by

$$
\eta_{C C, L}^{*} \leq \eta_{C C, \infty}^{*}=\frac{\kappa_{\infty} \theta_{\infty} \mathcal{T}_{0}}{4\left(2^{\mathcal{T}_{0}}-1\right)}
$$

where $\kappa_{\infty}=\lim _{L \rightarrow \infty} \kappa_{L} \approx 1.6617$ as proved in Appendix $F$. Particularly, for Rayleigh fading channels with unit channel gains as $\sigma_{1}{ }^{2}=\cdots=\sigma_{L}{ }^{2}=1, \theta_{\infty} \leq 1$ and the maximal energy efficiency of HARQ-CC with outage and goodput constraints satisfies

$\eta_{C C, \infty}^{*}=\frac{\kappa_{\infty} \theta_{\infty} \mathcal{T}_{0}}{4\left(2^{\mathcal{T}_{0}}-1\right)} \leq \lim _{\mathcal{T}_{0} \rightarrow 0} \frac{\kappa_{\infty} \mathcal{T}_{0}}{4\left(2^{\mathcal{T}_{0}}-1\right)}=\frac{\kappa_{\infty}}{4 \ln (2)} \triangleq \eta_{C C, \infty}^{\max }$

In other words, when QoS constraints are considered, the maximal energy efficiency of HARQ-CC is $\frac{\kappa_{\infty}}{4 \ln (2)}$ which is higher than that of Type I HARQ.

\section{HARQ-IR}

1) Optimal Rate: Putting (5) into (20) yields the energy efficiency of HARQ-IR as

$$
\eta_{I R, L}=\psi \theta_{L} \frac{f\left(\alpha^{*}\right) R}{\left(\prod_{k=1}^{L}\left(\frac{g_{k}(R)}{g_{k-1}(R)}\right)^{2^{-k}}\right)^{\frac{1}{1-2^{-L}}}} .
$$

Substituting (18) into (34), it becomes

$$
\begin{aligned}
\eta_{I R, L} & =\frac{\psi \theta_{L} \mathcal{T}_{0}}{\Lambda(R)}\left(\chi\left(R-\mathcal{T}_{0}\right)-\chi\left(R-\frac{\mathcal{T}_{0}}{1-\Delta}\right)\right) \\
+ & \frac{\psi \theta_{L}(1-\Delta) \Delta^{c}}{\left(\prod_{k=1}^{L}\left(\mathcal{G}_{k}(R)\right)^{2^{-k}}\right)^{\frac{1}{1-2^{-L}}}} \chi\left(R-\frac{\mathcal{T}_{0}}{1-\Delta}\right),
\end{aligned}
$$

where $\Lambda(R) \triangleq\left(1-\frac{\mathcal{T}_{0}}{R}\right)^{-c}\left(\prod_{k=1}^{L}\left(\frac{g_{k}(R)}{g_{k-1}(R)}\right)^{2^{-k}}\right)^{\frac{1}{1-2^{-L}}}$ and $\mathcal{G}_{k}(R) \triangleq \frac{g_{k}(R)}{R g_{k-1}(R)}$. When $R>\frac{\mathcal{T}_{0}}{1-\Delta}$, the first term in (35) is zero and the energy efficiency reduces as $\eta_{I R, L}=$

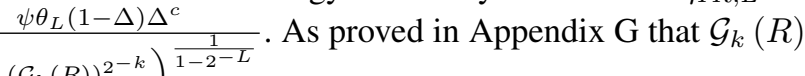
$\left(\prod_{k=1}^{L}\left(\mathcal{G}_{k}(R)\right)^{2-k}\right)^{\frac{1}{1-2}}$

is a monotonically increasing function of $R$. Thus when $R>\frac{\mathcal{T}_{0}}{1-\Delta}, \eta_{I R, L}$ is a decreasing function of $R$. Together with the continuity of $\eta_{I R, L}$ at the point $R=\frac{\mathcal{T}_{0}}{1-\Delta}$ and the constraint of $R>\mathcal{T}_{0}$ in (20), we can conclude that the optimal rate to achieve the maximal energy efficiency of HARQ-IR is 
located within the range of $\left(\mathcal{T}_{0}, \frac{\mathcal{T}_{0}}{1-\Delta}\right]$. Accordingly, when $\mathcal{T}_{0}<R \leq \frac{\mathcal{T}_{0}}{1-\Delta}$, the energy efficiency $\eta_{I R, L}$ can be simplified as

$$
\eta_{I R, L}=\frac{\psi \theta_{L} \mathcal{T}_{0}}{\Lambda(R)}
$$

Then the rate selection problem in (20) is equivalent to

$$
\begin{array}{ll}
\min _{R} & \Lambda(R) \\
\text { subject to } & \mathcal{T}_{0}<R \leq \frac{\mathcal{T}_{0}}{1-\Delta} .
\end{array}
$$

Due to the complicated form of $\Lambda(R)$, it is difficult to derive a closed-form solution for the optimal transmission rate. Fortunately, after analyzing the function $\Lambda(R)$, we find a special property of $\Lambda(R)$ in the following.

Property 2. The function $\Lambda(R)$ is bounded by

$$
\begin{gathered}
(\ln (2))^{\frac{2^{-1}-2^{-L}}{1-2^{-L}}} \kappa_{L}-\frac{1}{1-2^{-L}}(\varpi(R))^{\frac{2^{-1}}{1-2^{-L}}} \leq \Lambda(R) \\
\leq(\ln (2))^{\frac{2^{-1}-2^{-L}}{1-2^{-L}}} \kappa_{L-1}-\frac{2^{-1}}{1-2^{-L}}(\varpi(R))^{\frac{2^{-1}}{1-2^{-L}}}, \\
\text { where } \varpi(R)=\left(R-\mathcal{T}_{0}\right)^{-2^{-L+1}}\left(2^{R}-1\right) R .
\end{gathered}
$$

Proof: Please see Appendix H.

With these bounds and Intermediate Value Theorem [35, Theorem 4.23], $\Lambda(R)$ can be rewritten as

$$
\Lambda(R)=(\ln (2))^{\frac{2^{-1}-2^{-L}}{1-2^{-L}}} \zeta(\varpi(R))^{\frac{2^{-1}}{1-2^{-L}}},
$$

where $\zeta$ is bounded as

$$
\kappa_{L}^{-\frac{1}{1-2^{-L}}} \leq \zeta \leq \kappa_{L-1}-\frac{2^{-1}}{1-2^{-L}}
$$

Based on (39), the optimization problem (37) can be rewritten as

$$
\begin{array}{ll}
\min _{R} & \varpi(R) \\
\text { subject to } & \mathcal{T}_{0}<R \leq \frac{\mathcal{T}_{0}}{1-\Delta} .
\end{array}
$$

Following a similar proof as that for Theorem 2, the optimal solution to (41) can be derived by using KKT conditions, as given in the following theorem.

Theorem 5. The optimal rate to the problem in (41) is

$$
R^{*}=\min \left\{\frac{\mathcal{T}_{0}}{1-\Delta}, \Upsilon^{-1}(0)\right\}
$$

where $\Upsilon(R)=\left(R-\mathcal{T}_{0}\right)\left(2^{R} \ln (2) R+2^{R}-1\right)-$ $2^{-L+1}\left(2^{R}-1\right) R$ and $\Upsilon^{-1}(0)$ refers to the zero point of $\Upsilon(R)$. When $\varepsilon \geq 2^{-L}$, the optimal transmission rate reduces to $R^{*}=\Upsilon^{-1}(0)$.

The proof is omitted here to avoid redundancy. Notice that the zero point $\Upsilon^{-1}(0)$ can be easily found since $\Upsilon(R)$ is an increasing function with respect to $R$.

2) Optimal Energy Efficiency: With the definition of $\Lambda(R)$, the energy efficiency of HARQ-IR in (36) can be written as

$$
\eta_{I R, L}=\frac{\psi \theta_{L} \mathcal{T}_{0}}{\left(1-\frac{\mathcal{T}_{0}}{R}\right)^{-c}\left(\prod_{k=1}^{L}\left(\frac{g_{k}(R)}{g_{k-1}(R)}\right)^{2-k}\right)^{\frac{1}{1-2^{-L}}}}
$$

As proved in Appendix I, we have the following inequality

$$
\frac{g_{k}(R)}{g_{k-1}(R)} \leq \frac{2^{R}-1}{k} \text {. }
$$

Applying this inequality to (43) yields

$$
\begin{aligned}
\eta_{I R, L} & \geq \frac{\left(\prod_{k=1}^{L} k^{2^{-k}}\right)^{\frac{1}{1-2^{-L}}} \psi \theta_{L} \mathcal{T}_{0}\left(1-\frac{\mathcal{T}_{0}}{R}\right)^{c}}{\left(\prod_{k=1}^{L}\left(2^{R}-1\right)^{2^{-k}}\right)^{\frac{1}{1-2^{-L}}}} \\
& =\kappa_{L} \frac{1}{1-2^{-L}} \psi \theta_{L} \mathcal{T}_{0} \frac{\left(1-\frac{\mathcal{T}_{0}}{R}\right)^{c}}{2^{R}-1}=\eta_{C C, L} .
\end{aligned}
$$

It means that HARQ-IR outperforms HARQ-CC in terms of energy efficiency. Moreover, the optimal energy efficiency of HARQ-IR is also higher than or equal to that of HARQ-CC, i.e., $\eta_{I R, L}^{*} \geq \eta_{C C, L}^{*}$.

Now putting the optimal rate (42) into (39) and then (36) together with the bounds of $\zeta$ in (40), the optimal energy efficiency of HARQ-IR is bounded as

$$
\kappa_{L-1} \frac{2^{-1}}{1-2^{-L}} \tilde{\eta}_{I R, L}^{*} \leq \eta_{I R, L}^{*} \leq \kappa_{L}^{\frac{1}{1-2^{-L}}} \tilde{\eta}_{I R, L}^{*} .
$$

where

$$
\tilde{\eta}_{I R, L}^{*}=\psi \theta_{L} \mathcal{T}_{0}(\ln (2))^{-\frac{2^{-1}-2^{-L}}{1-2^{-L}}}\left(\varpi\left(R^{*}\right)\right)^{-\frac{2^{-1}}{1-2^{-L}}} .
$$

Combining (46) with the inequality $\eta_{I R, L}^{*} \geq \eta_{C C, L}^{*}$, the optimal energy efficiency of HARQ-IR is consequently found to be bounded by

$\max \left\{\kappa_{L-1} \frac{2^{-1}}{1-2^{-L}} \tilde{\eta}_{I R, L}^{*}, \eta_{C C, L}^{*}\right\} \leq \eta_{I R, L}^{*} \leq \kappa_{L^{\frac{1}{1-2^{-L}}}}^{\tilde{\eta}_{I R, L}^{*}}$.

When the number of transmissions approaches infinity, i.e., $L \rightarrow \infty$, the following bounds also hold

$$
\max \left\{\sqrt{\kappa_{\infty}} \tilde{\eta}_{I R, \infty}^{*}, \eta_{C C, \infty}^{*}\right\} \leq \eta_{I R, \infty}^{*} \leq \kappa_{\infty} \tilde{\eta}_{I R, \infty}^{*},
$$

where $\eta_{C C, \infty}^{*}$ has been given in Theorem 4 , and $\tilde{\eta}_{I R, \infty}^{*}$ is defined as $\tilde{\eta}_{I R, \infty}^{*} \triangleq \lim _{L \rightarrow \infty} \tilde{\eta}_{I R, L}^{*}$ and can be further derived from (47) as

$$
\begin{aligned}
& \tilde{\eta}_{I R, \infty}^{*} \\
& =\lim _{L \rightarrow \infty} \psi \theta_{L} \mathcal{T}_{0}(\ln (2))^{-\frac{2^{-1}-2^{-L}}{1-2^{-L}}} \\
& \quad \times\left(\left(R^{*}-\mathcal{T}_{0}\right)^{-2^{-L+1}}\left(2^{R^{*}}-1\right) R^{*}\right)^{-\frac{2^{-1}}{1-2^{-L}}} \\
& =\frac{\theta_{\infty} \mathcal{T}_{0}}{4 \sqrt{\ln (2)}} \lim _{L \rightarrow \infty}\left(\left(R^{*}-\mathcal{T}_{0}\right)^{-2^{-L+1}}\left(2^{R^{*}}-1\right) R^{*}\right)^{-\frac{2^{-1}}{1-2^{-L}}}
\end{aligned}
$$

In (50), the last equality follows based on the limit result $\lim _{L \rightarrow \infty} \psi=\frac{1}{4}$.

When $L \rightarrow \infty$, the inequality $\varepsilon \geq 2^{-L}$ would hold. Based on Theorem 5, the optimal transmission rate is $R^{*}=\Upsilon^{-1}(0)$ and thus $\Upsilon\left(R^{*}\right)=0$ which can be rewritten as

$$
R^{*}=\mathcal{T}_{0}+\frac{2^{-L+1}\left(2^{R^{*}}-1\right) R^{*}}{2^{R^{*}} \ln (2) R^{*}+2^{R^{*}}-1} .
$$


Meanwhile, noticing that the optimal rate $R^{*}$ is bounded as $\mathcal{T}_{0}<R^{*} \leq \frac{\mathcal{T}_{0}}{1-\Delta}, \Delta=\min \left\{\varepsilon, 2^{-L}\right\}$ and $\lim _{L \rightarrow \infty} \Delta=0$, it follows by using squeeze theorem that $\lim _{L \rightarrow \infty} R^{*}=\mathcal{T}_{0}$. With this limit and plugging (51) into (50), it yields

$$
\begin{aligned}
& \tilde{\eta}_{I R, \infty}^{*} \\
& =\frac{\theta_{\infty} \mathcal{T}_{0}}{4 \sqrt{\ln (2)}} \lim _{L \rightarrow \infty}\left(\left(2^{R^{*}}-1\right) R^{*}\right)^{-\frac{2^{-1}}{1-2^{-L}}} \lim _{L \rightarrow \infty}\left(R^{*}-\mathcal{T}_{0}\right) \\
& =\frac{\theta_{\infty}}{4} \sqrt{\frac{\mathcal{T}_{0}}{\ln (2)\left(2^{\mathcal{T}_{0}}-1\right)}} \lim _{L \rightarrow \infty}\left(\frac{2^{-L+1}\left(2^{R^{*}}-1\right) R^{*}}{2^{R^{*}} \ln (2) R^{*}+2^{R^{*}}-1}\right)^{\frac{2}{1-2}} \\
& =\frac{\theta_{\infty}}{4} \sqrt{\frac{\mathcal{T}_{0}}{\ln (2)\left(2^{\mathcal{T}_{0}}-1\right)}} . \\
& \max \left\{\frac{\theta_{\infty}}{4} \sqrt{\frac{\kappa_{\infty} \mathcal{T}_{0}}{\ln (2)\left(2^{\mathcal{T}_{0}}-1\right)}}, \frac{\kappa_{\infty} \theta_{\infty} \mathcal{T}_{0}}{4\left(2^{\mathcal{T}_{0}}-1\right)}\right\} \leq \eta_{I R, \infty}^{*} \\
& \leq \frac{\kappa_{\infty} \theta_{\infty}}{4} \sqrt{\frac{\mathcal{T}_{0}}{\ln (2)\left(2^{\mathcal{T}_{0}}-1\right)}}
\end{aligned}
$$

Clearly, both the lower and upper bounds of $\eta_{I R, \infty}^{*}$ in (53) are decreasing functions of the the goodput threshold $\mathcal{T}_{0}$. In addition, the following inequality of their limits holds

$$
\begin{array}{r}
\max \left\{\frac{\theta_{\infty}}{4} \sqrt{\frac{\kappa_{\infty}}{\ln (2)}} \sqrt{\lim _{\mathcal{T}_{0} \rightarrow 0} \frac{\mathcal{T}_{0}}{2^{\mathcal{T}_{0}}-1}}, \frac{\kappa_{\infty} \theta_{\infty}}{4} \lim _{\mathcal{T}_{0} \rightarrow 0} \frac{\mathcal{T}_{0}}{2^{\mathcal{T}_{0}}-1}\right\} \\
\leq \lim _{\mathcal{T}_{0} \rightarrow 0} \eta_{I R, \infty}^{*} \leq \frac{\kappa_{\infty} \theta_{\infty}}{4 \sqrt{\ln (2)}} \sqrt{\lim _{\mathcal{T}_{0} \rightarrow 0} \frac{\mathcal{T}_{0}}{2^{\mathcal{T}_{0}}-1}} .
\end{array}
$$

Noticing that $\lim _{\mathcal{T}_{0} \rightarrow 0} \frac{\mathcal{T}_{0}}{2^{T_{0}-1}}=\frac{1}{\ln (2)}$, we have

$$
\max \left\{\frac{\sqrt{\kappa_{\infty}} \theta_{\infty}}{4 \ln (2)}, \frac{\kappa_{\infty} \theta_{\infty}}{4 \ln (2)}\right\} \leq \lim _{\mathcal{T}_{0} \rightarrow 0} \eta_{I R, \infty}^{*} \leq \frac{\kappa_{\infty} \theta_{\infty}}{4 \ln (2)} .
$$

Since $\kappa_{\infty}>1$ and using squeeze theorem, it follows

$$
\lim _{\mathcal{T}_{0} \rightarrow 0} \eta_{I R, \infty}^{*}=\frac{\kappa_{\infty} \theta_{\infty}}{4 \ln (2)} .
$$

Summarizing the results in (53), (56) and Property 1 finally leads to the following result of the optimal energy efficiency of HARQ-IR.

Theorem 6. Under time-correlated Rayleigh fading channels, the optimal energy efficiency of HARQ-IR with outage and goodput constraints is upper bounded by

$$
\eta_{I R, L}^{*} \leq \eta_{I R, \infty}^{*} \leq \frac{\kappa_{\infty} \theta_{\infty}}{4} \sqrt{\frac{\mathcal{T}_{0}}{\ln (2)\left(2^{\mathcal{T}_{0}}-1\right)}} .
$$

Particularly, for Rayleigh fading channels with unit channel gains $\sigma_{1}{ }^{2}=\cdots=\sigma_{L}{ }^{2}=1, \theta_{\infty} \leq 1$ and the maximal energy efficiency of HARQ-IR with outage and goodput constraints follows

$$
\eta_{I R, \infty}^{*} \leq \frac{\kappa_{\infty} \theta_{\infty}}{4} \sqrt{\frac{\mathcal{T}_{0}}{\ln (2)\left(2^{\mathcal{T}_{0}}-1\right)}} \leq \frac{\kappa_{\infty}}{4 \ln (2)} \triangleq \eta_{I R, \infty}^{\max }
$$

Comparing the results in Theorems 4 and 6 , we can conclude that HARQ-CC and HARQ-IR can reach the same maximal energy efficiency of $\frac{\kappa_{\infty}}{4 \ln (2)}$.

\section{Numerical Results and Discussions}

The performance of our proposed energy-efficient optimiza-

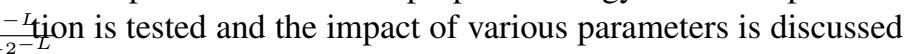
in this section. Unless otherwise stated, the results are provided ${ }_{L}$ over time-correlated Rayleigh fading channels with time corFelation $\rho=0.5$ and unit channel gains $\sigma_{1}^{2}=\cdots=\sigma_{L}^{2}=1$.

\section{A. Numerical Verification}

In our design, asymptotic outage probability is adopted to enable the derivation of closed-form solutions for the optimal transmission powers and the optimal rate. To validate the correctness of our design, the optimal energy efficiency achieved by our design is compared with that achieved through numerical exhaustive search based on the exact outage probabilities derived in [28]-[30]. The results versus the outage tolerance $\varepsilon$ and the minimum goodput requirement $\mathcal{T}_{0}$ are shown in Figs. 1 and 2, respectively. It is readily observed from Fig. 1 that there is an excellent match between the optimal energy efficiencies based on the asymptotic and the exact outage probabilities when $\varepsilon \leq 10^{-2}$, because the outage probability can be well approximated by the asymptotic outage probability (4) under a low outage (or high SNR). This result demonstrates that the effectiveness of the proposed closeform solution holds true when outage constraint is strict, i.e., the allowable outage probability is small, which is usually true in practical applications since the high QoS is generally required in practice. The effectiveness of our design is further demonstrated by the results in Fig. 2 where the optimal energy efficiency achieved by our design coincides well with that achieved based on the exact outage probability no matter how large the minimum goodput. Moreover, it is also shown in Fig. 1 that the optimal energy efficiency $\eta_{L}^{*}$ is an increasing function of $\varepsilon$. For instance, $\eta_{L}^{*}$ of HARQ-IR scheme is increased by about 0.15 bits/J when the outage tolerance is relaxed from $10^{-3}$ to $10^{-1}$. However, as shown in Fig. 2, the optimal energy efficiency decreases with the increase of the minimum goodput requirement $\mathcal{T}_{0}$, which verifies our result that the maximal energy efficiency is achieved when no goodput constraint is considered, i.e., $\mathcal{T}_{0} \rightarrow 0$. In addition, both Figs. 1 and 2 show that HARQ-IR performs the best in terms of optimal energy efficiency, while Type I HARQ provides the worst performance without exploiting the erroneously received subcodewords.

To further demonstrate the superiority of our design, our energy-efficient design is compared with uniform power design and their optimal energy efficiencies are shown in Fig. 3 . Notice that in the uniform power design, the transmission powers at different HARQ rounds are set equal, i.e., $P_{1}=$ $\cdots=P_{L}=P$, and the transmission power $P$ and the rate are then optimally chosen to maximize the energy efficiency under the same QoS constraints as our design. Clearly, our design can achieve a significant enhancement of energy efficiency over the uniform power design under a stringent outage constraint. 


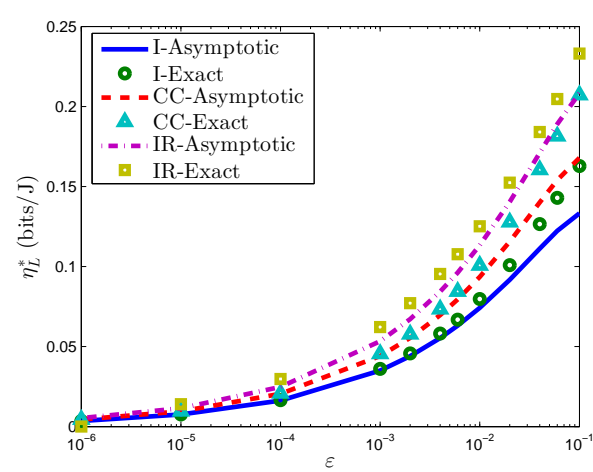

Fig. 1. The optimal energy efficiency versus the outage tolerance with $L=2$ and $\mathcal{T}_{0}=2 \mathrm{bps} / \mathrm{Hz}$

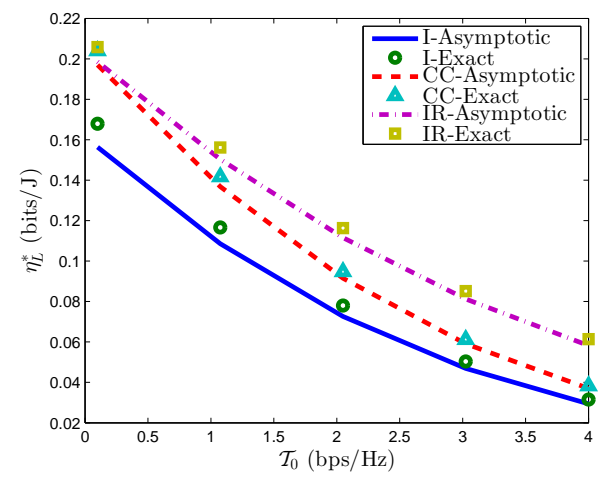

Fig. 2. The optimal energy efficiency versus the minimum goodput requirement with $L=2$ and $\varepsilon=10^{-2}$.

Interestingly, for Type I HARQ, the energy efficiency achieved by the proposed design converges to that of the uniform power design under loose outage constraint, i.e., $\varepsilon \rightarrow 1$. However, there is a non-negligible gap between the energy efficiencies of the proposed design and the uniform power design for both HARQ-CC and HARQ-IR. This is due to the fact that the erroneously received packets are directly discarded by Type I HARQ and thus the previously consumed resources such as transmission powers are not exploited. To overcome this problem, both HARQ-CC and HARQ-IR combine the currently received packet with the erroneously received packets for reutilizing these resources. Particularly for HARQ-CC and HARQIR, the concluded results are totally different from [36], where the uniform power allocation can offer similar performance as the optimal solution for spectral efficiency maximization. This essentially stems from the difference between the energy and the spectral efficiencies. The energy efficiency is in fact a ratio of spectral efficiency to the long term average power [37], so it would be significantly affected by transmission powers. As opposed to [18, Fig. 3], [36] where the spectral efficiency maximization is targeted and only a slight spectral efficiency improvement can be achieved via optimal power allocation, the adaptation of transmission powers appears to be very crucial to the energy efficiency maximization.

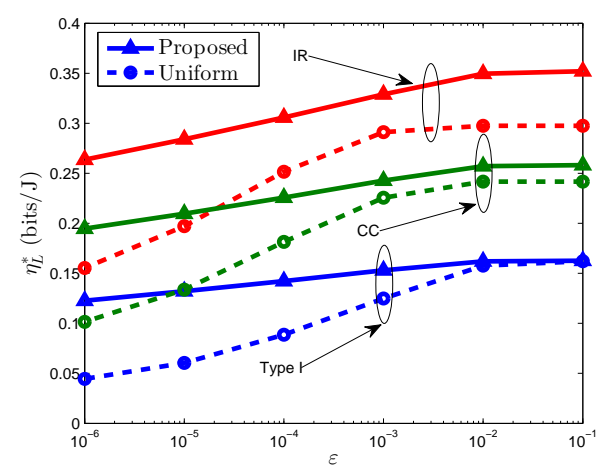

Fig. 3. Comparison of the proposed design with uniform power design for various HARQ schemes by setting $\mathcal{T}_{0}=2 \mathrm{bps} / \mathrm{Hz}$ and $L=5$.

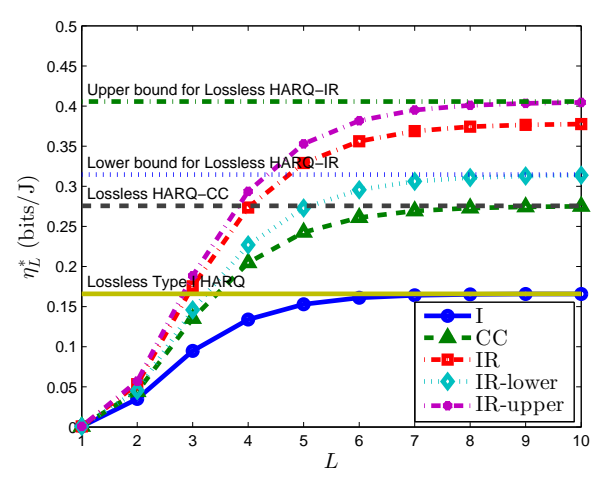

Fig. 4. The optimal energy efficiency versus the maximal number of transmissions with $\mathcal{T}_{0}=2 \mathrm{bps} / \mathrm{Hz}$ and $\varepsilon=10^{-4}$.

\section{B. Impact of System Parameters}

To investigate the impact of various system parameters, the optimal energy efficiency versus the maximal number of transmissions is shown in Fig. 4. It is clear that the optimal energy efficiency $\eta_{L}^{*}$ is an increase function of $L$ and is upper bounded. The maximal energy efficiency is achieved when $L \rightarrow \infty$, which is named as lossless HARQ for convenience. This is consistent with our analytical results in Theorems 3,4 and 6 . Since the energy efficiency is upper bounded, when $L$ is large, further increase of the maximal number of transmission can only contribute a very limited improvement on the energy efficiency, but it would definitely lead to the reduction of spectral efficiency since more transmissions are conducted to convey the same information. Thus there would exist a tradeoff between the energy efficiency and the spectral efficiency, which will be particularly discussed later. Herein, it should be pointed out that $\theta_{\infty}$ is a function of $\rho$, and can be approximated as $\theta_{L}$ with high accuracy by choosing $L=20$.

To test the effect of the outage tolerance $\varepsilon$, the optimal energy efficiency $\eta_{L}^{*}$ versus $\varepsilon$ is plotted in Fig. 5. It is found that the optimal energy efficiency first increases as $\varepsilon$ increases up to 0.06 , while it remains constant when $\varepsilon$ increases further. This result can be well explained by Theorem 2 and Theorem 5. More precisely, when $\varepsilon>2^{-L}$, the optimal rate is independent of the outage tolerance $\varepsilon$ and thus the optimal energy efficiency becomes irrelevant to $\varepsilon$. In the case of $L=4$, we have $2^{-L}=2^{-4} \approx 0.06$. Therefore, when $\varepsilon>0.06$, the optimal energy efficiency would become constant in this case. 


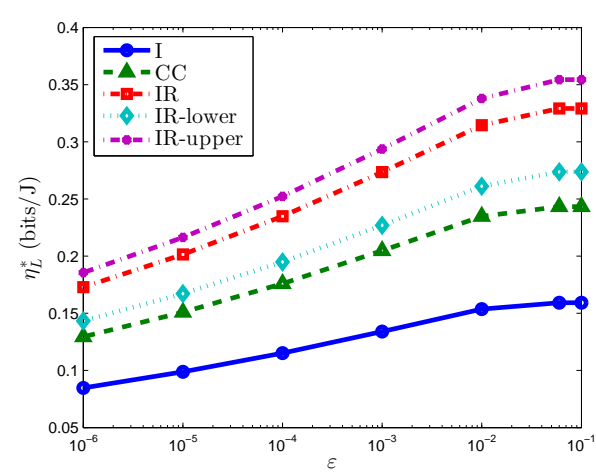

Fig. 5. The optimal energy efficiency versus the outage tolerance with $\mathcal{T}_{0}=2$ bps/Hz and $L=4$.

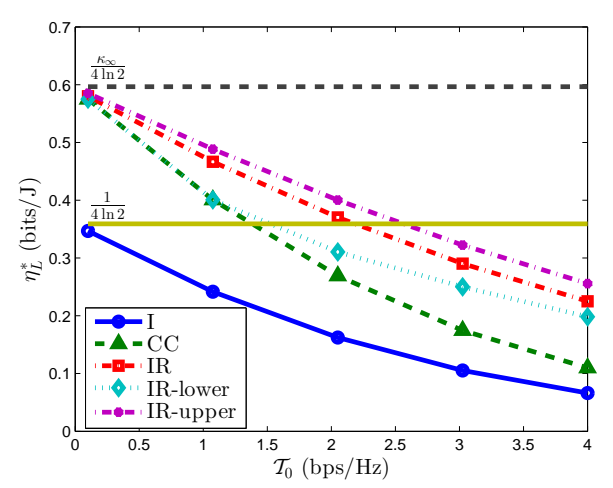

Fig. 6. The optimal energy efficiency versus the minimum goodput requirement with $L=10$ and $\varepsilon=10^{-2}$.

The effect of the minimal goodput requirement $\mathcal{T}_{0}$ is then shown in Fig. 6. It can be seen that the optimal energy efficiency $\eta_{L}^{*}$ decreases with the increase of $\mathcal{T}_{0}$. For all the three HARQ schemes, the maximal energy efficiency is achieved when $\mathcal{T}_{0} \rightarrow 0$. Moreover, HARQ-CC and HARQIR can achieve the same maximal energy efficiency of $\frac{\kappa_{\infty}}{4 \ln (2)}$ when $\mathcal{T}_{0} \rightarrow 0$. However, the superiority of HARQ-IR over HARQ-CC becomes more significant as $\mathcal{T}_{0}$ increases.

The impact of the time correlation $\rho$ on the optimal energy efficiency $\eta_{L}^{*}$ is finally investigated and the results are shown in Fig. 7. As proved in Appendix $C, \theta_{L}$ is a decreasing function of the time correlation $\rho$ and thus the time correlation has a detrimental impact on the energy efficiency. This can be verified by the results in Fig. 7. It can also been seen that there is a significant drop of the energy efficiency when $\rho>0.5$, which is consistent with the result in [38] that time correlation lower than 0.5 would not lead to a significant performance degradation.

\section{Spectral Efficiency}

As defined in [14], [16], spectral efficiency of HARQ schemes signifies the average number of successfully delivered bits per channel use $(\mathrm{bps} / \mathrm{Hz})$ and is defined as

$$
\xi_{L}=\frac{R\left(1-p_{\text {out }, L}\right)}{\sum_{l=0}^{L-1} p_{\text {out }, l}} .
$$

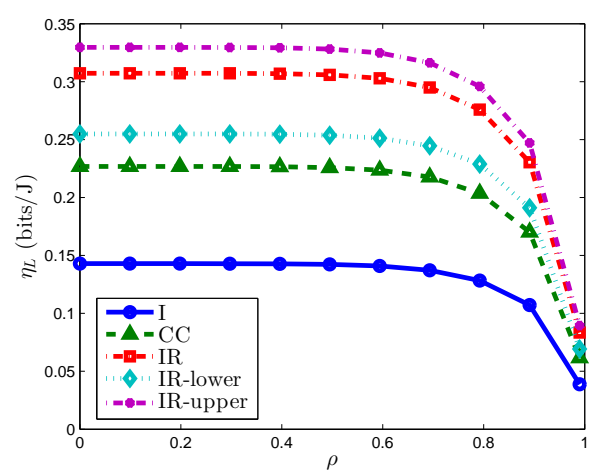

Fig. 7. Effect of time correlation with $L=5, \mathcal{T}_{0}=2 \mathrm{bps} / \mathrm{Hz}$ and $\varepsilon=10^{-4}$.

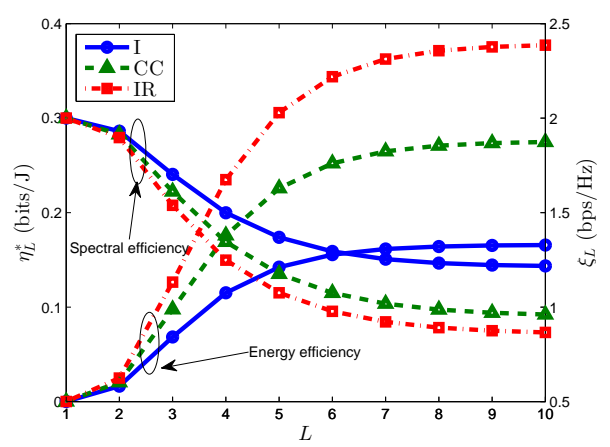

Fig. 8. Spectral and energy efficiencies of various HARQ schemes with $\mathcal{T}_{0}=2 \mathrm{bps} / \mathrm{Hz}$ and $\varepsilon=10^{-4}$.

Generally, the optimizations of the energy efficiency and the spectral efficiency are two conflicting goals. This conflict can be clearly observed from the results of energy and spectral efficiencies achieved by our design as shown in Fig. 8. It is shown that the spectral efficiency $\xi_{L}$ decreases while the optimal energy efficiency $\eta_{L}^{*}$ increases with the increase of $L$ and higher energy efficiency is achieved at the cost of spectral efficiency degradation. In addition, with our energyefficient optimization, the HARQ-IR scheme can achieve the highest energy efficiency but with the lowest spectral efficiency since our design is targeting at energy efficiency maximization. Notice that this result is different from that in [5], [9], [10] where the design objective is to maximize the spectral efficiency. To further illustrate the tradeoff between the energy and spectral efficiencies for the three HARQ schemes, the minimum goodput requirement $\mathcal{T}_{0}$ is varied from $0.5 \mathrm{bps} / \mathrm{Hz}$ to $10 \mathrm{bps} / \mathrm{Hz}$ and the corresponding efficiencies are shown in Fig. 9. It is clear from Figs. 8 and 9 that HARQ-CC can always achieve spectral and energy efficiencies in between those of Type I HARQ and HARQ-IR given the same objective and constraints for optimization. In other words, HARQ-CC can achieve a better tradeoff between the energy and spectral efficiencies than the other schemes given the same objective and constraints for optimization.

To demonstrate the generality of the above results, a practical long term evolution (LTE) system with a coding rate of $1 / 2$ and a modulation scheme of 16QAM is also taken as an example for simulations. The system level simulation is conducted by using LTE system toolbox for MATLAB. 


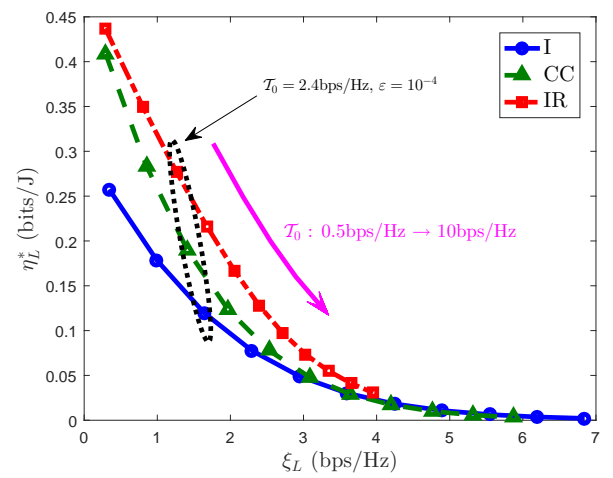

Fig. 9. Tradeoff between the optimal energy efficiency and spectral efficiency with $\varepsilon=10^{-4}$ and $L=5$.

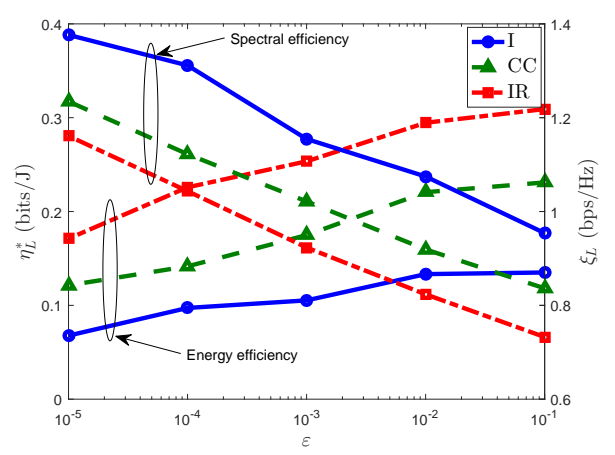

Fig. 10. Spectral and energy efficiencies for the three HARQ schemes in LTE system with a coding rate of $1 / 2$, a modulation scheme of 16QAM, $N_{b}=12960$ bits and $L=4$.

Note that since $\mathcal{T}_{0}<R \leq \frac{\mathcal{T}_{0}}{1-\Delta}$ and $\Delta$ is small, $\mathcal{T}_{0}$ can be approximated as $\mathcal{T}_{0} \approx R=1 / 2 \times \log _{2} 16=2 \mathrm{bps} / \mathrm{Hz}$. Hereby, by using the closed-form solution of problem (9) with $\mathcal{T}_{0}=2$ bps/Hz, Fig. 10 shows the spectral and the energy efficiencies of the three HARQ schemes against the outage constraint under LTE system settings. Similar results can be observed as Fig. 8, that is, HARQ-CC can strike the best balance between the energy and the spectral efficiencies.

\section{CONCLUSION}

Energy-efficient optimization for HARQ schemes has been proposed in this paper. Different from the prior designs, widely occurred time-correlated fading channels and practical QoS constraints are considered in the optimal design to maximize the energy efficiency. The optimal transmission powers and the optimal rate have been derived in closed-forms and have further enabled the analysis of the maximal energy efficiencies of various HARQ schemes. It has been found that with low outage constraint, the maximal energy efficiencies of Type I HARQ and HARQ-CC/IR are $\frac{1}{4 \ln 2}$ bits/J and $\frac{\kappa_{\infty}}{4 \ln 2}$ bits/J, respectively. Our numerical results have also shown that the energy efficiency improvement is achieved in sacrifice of the spectral efficiency, because the spectral efficiency and the energy efficiency are two conflicting objectives. In addition, HARQ-CC can achieve a better tradeoff between the energy efficiency and the spectral efficiency than Type I HARQ and HARQ-IR.

\section{APPENDIX A \\ PROOF OF THEOREM 1}

It is clear from (12) that the target outage probability $\alpha$ should be nonzero, i.e., $\alpha \neq 0$. Together with the first constraint in (17), we have $0<\alpha \leq \varepsilon$. Further considering the second constraint $R(1-\alpha) \geq \overline{\mathcal{T}}_{0}$, the transmission rate should satisfy $R>\mathcal{T}_{0}$, otherwise the second constraint cannot be satisfied and the problem (17) is then infeasible. Then the problem (17) can be reformulated as

$$
\begin{array}{ll}
\max _{\alpha} & f(\alpha) \\
\text { subject to } & 0<\alpha \leq \min \left\{\varepsilon, 1-\frac{\mathcal{T}_{0}}{R}\right\} .
\end{array}
$$

The Lagrangian associated with (60) can be written as

$$
\mathcal{L}_{1}(\alpha, u)=f(\alpha)+u\left(\alpha-\min \left\{\varepsilon, 1-\frac{\mathcal{T}_{0}}{R}\right\}\right)-\nu \alpha,
$$

where $u$ and $\nu$ refer to Lagrange multipliers. The optimal solution to (60) should satisfy the Karush-Khun-Tucker (KKT) conditions as

$$
\begin{gathered}
\left.\frac{\partial \mathcal{L}_{1}}{\partial \alpha}\right|_{\left(\alpha^{*}, u^{*}, \nu^{*}\right)}=f^{\prime}\left(\alpha^{*}\right)+u^{*}-\nu^{*}=0, \\
u^{*}\left(\alpha^{*}-\min \left\{\varepsilon, 1-\frac{\mathcal{T}_{0}}{R}\right\}\right)=0, \\
\nu^{*} \alpha^{*}=0,
\end{gathered}
$$

$0<\alpha^{*} \leq \min \left\{\varepsilon, 1-\frac{\mathcal{T}_{0}}{R}\right\}$ and $u^{*}, \nu^{*} \geq 0$. Since $\alpha^{*}>0$, (64) implies $\nu^{*}=0$. Substituting $\nu^{*}=0$ into (62) yields

$$
u^{*}=-f^{\prime}\left(\alpha^{*}\right)=\frac{\alpha^{* \frac{1}{2^{L}}-1}}{2^{L}-1}\left(\alpha^{*-1}-2^{L}\right) .
$$

Note that the Lagrange multiplier $u^{*}$ is either larger than or equal to 0 . Suppose that $u^{*}=0$, it follows from (65) that $\alpha^{*}=2^{-L}$. Combining this with (63) and the constraint $0<\alpha^{*} \leq \min \left\{\varepsilon, 1-\frac{\mathcal{T}_{0}}{R}\right\}$, we have $\alpha^{*}=2^{-L} \leq$ $\min \left\{\varepsilon, 1-\frac{\mathcal{T}_{0}}{R}\right\}$. If $u^{*}>0$, on the other hand, (63) implies that $\alpha^{*}=\min \left\{\varepsilon, 1-\frac{\mathcal{T}_{0}}{R}\right\}$. Besides, combining $u^{*}>0$ with (65) gives $\alpha^{*-1}-2^{L}>0$ so that $\alpha^{*}=\min \left\{\varepsilon, 1-\frac{\mathcal{T}_{0}}{R}\right\}<$ $2^{-L}$. To summarize, the optimal target outage probability is therefore given by $\alpha^{*}=\min \left\{\varepsilon, 1-\frac{\mathcal{T}_{0}}{R}, 2^{-L}\right\}$ for $R>\mathcal{T}_{0}$.

By defining $\Delta=\min \left\{\varepsilon, 2^{-L}\right\}$, the optimal target outage probability $\alpha^{*}$ can be rewritten as

$$
\begin{aligned}
& \alpha^{*}=\min \left\{\Delta, 1-\frac{\mathcal{T}_{0}}{R}\right\}=\left(1-\frac{\mathcal{T}_{0}}{R}\right) \times \\
& \left(\chi\left(R-\mathcal{T}_{0}\right)-\chi\left(R-\frac{\mathcal{T}_{0}}{1-\Delta}\right)\right)+\Delta \chi\left(R-\frac{\mathcal{T}_{0}}{1-\Delta}\right),
\end{aligned}
$$

where $\chi(\cdot)$ denotes the indicator function as (19). Plugging (66) into $f(\alpha)=(1-\alpha) \alpha^{\frac{1}{2^{L}-1}}$ finally leads to (18). 


\section{APPENDIX B}

\section{Proof of THEOREM 2}

The Lagrangian associated with (25) is written as

$$
\mathcal{L}_{2}(R, v, w)=\Phi(R)+v\left(\mathcal{T}_{0}-R\right)+w\left(R-\frac{\mathcal{T}_{0}}{1-\Delta}\right)
$$

where $v$ and $w$ represent the Lagrange multipliers. The optimal solution to (25) should satisfy the KKT conditions as

$$
\begin{gathered}
\left.\frac{\partial \mathcal{L}_{2}}{\partial R}\right|_{\left(R^{*}, v^{*}, w^{*}\right)}=\Phi^{\prime}\left(R^{*}\right)-v^{*}+w^{*}=0, \\
v^{*}\left(\mathcal{T}_{0}-R^{*}\right)=0, \\
w^{*}\left(R^{*}-\frac{\mathcal{T}_{0}}{1-\Delta}\right)=0,
\end{gathered}
$$

$\mathcal{T}_{0}<R^{*} \leq \frac{\mathcal{T}_{0}}{1-\Delta}$ and $v^{*}, w^{*} \geq 0$. Herein, the first derivative of $\Phi(R)$ with respect to $R$ is

$$
\Phi^{\prime}(R)=\frac{1}{R^{1-c}\left(R-\mathcal{T}_{0}\right)^{c+1}} \varphi(R),
$$

where $\varphi(R)=\ln (2) R\left(R-\mathcal{T}_{0}\right) 2^{R}-c \mathcal{T}_{0}\left(2^{R}-1\right)$. Noticing $\mathcal{T}_{0}<R^{*}$, it follows from (69) that $v^{*}=0$. Clearly from (68), $w^{*}$ is thus given by

$$
w^{*}=-\Phi^{\prime}\left(R^{*}\right)=-\frac{1}{R^{* 1-c}\left(R^{*}-\mathcal{T}_{0}\right)^{c+1}} \varphi\left(R^{*}\right) .
$$

Note that $w^{*}$ is either larger than or equal to 0 . If $w^{*}>0$, it follows from (70) that $R^{*}=\frac{\mathcal{T}_{0}}{1-\Delta}$. Together with (72), we have $\varphi\left(R^{*}\right)=\varphi\left(\frac{\mathcal{T}_{0}}{1-\Delta}\right)<0$. On the other hand, if $w^{*}=0$, following from (72), we have $\varphi\left(R^{*}\right)=0$. By defining $\varphi^{-1}(y)$ as the inverse function of $\varphi$, we have $R^{*}=\varphi^{-1}(0)$. Since $R>\mathcal{T}_{0}$, the following inequality holds

$$
\varphi^{\prime}(R)=\ln (2) 2^{R}\left(\ln (2) R\left(R-\mathcal{T}_{0}\right)+2 R-(1+c) \mathcal{T}_{0}\right)>0 .
$$

It means that $\varphi(R)$ is a monotonically increasing function of the rate $R$. Together with $R^{*} \leq \frac{\mathcal{T}_{0}}{1-\Delta}$, we have $\varphi\left(\frac{\mathcal{T}_{0}}{1-\Delta}\right) \geq$ $\varphi\left(R^{*}\right)=0$. Then the optimal solution $R^{*}$ under the above two cases can be summarized as

$$
R^{*}=\left\{\begin{array}{cc}
\frac{\mathcal{T}_{0}}{1-\Delta}, & \varphi\left(\frac{\mathcal{T}_{0}}{1-\Delta}\right)<0 \\
\varphi^{-1}(0), & \varphi\left(\frac{\mathcal{T}_{0}}{1-\Delta}\right) \geq 0 .
\end{array}\right.
$$

Noticing that $\varphi(R)$ is an increasing function of $R$, (74) can be rewritten as (26).

Clearly from (74), the optimal rate $R^{*}$ is determined by the sign of $\varphi\left(\frac{\mathcal{T}_{0}}{1-\Delta}\right)$. Based on its definition, $\varphi\left(\frac{\mathcal{T}_{0}}{1-\Delta}\right)$ can be explicitly expressed as

$\varphi\left(\frac{\mathcal{T}_{0}}{1-\Delta}\right)=2^{\frac{\mathcal{T}_{0}}{1-\Delta}} \mathcal{T}_{0}\left(\ln (2) \frac{\Delta \mathcal{T}_{0}}{(1-\Delta)^{2}}+c\left(2^{-\frac{\tau_{0}}{1-\Delta}}-1\right)\right)$

By using the inequality $2^{-x} \geq 1-x \ln 2$, we have

$$
\varphi\left(\frac{\mathcal{T}_{0}}{1-\Delta}\right) \geq 2^{\frac{\mathcal{T}_{0}}{1-\Delta}} \mathcal{T}_{0} \ln (2) \frac{\mathcal{T}_{0}}{1-\Delta}\left(\frac{\Delta}{1-\Delta}-c\right) .
$$

When $\varepsilon \geq 2^{-L}, \Delta=\min \left\{\varepsilon, 2^{-L}\right\}=2^{-L}$ and the right hand side of (76) equals to 0 since $c=\frac{1}{2^{L}-1}$. In other words, when $\varepsilon \geq 2^{-L}$, we have $\varphi\left(\frac{\mathcal{T}_{0}}{1-\Delta}\right) \geq 0$. From (74), the optimal transmission rate is thus given by $R^{*}=\varphi^{-1}(0)$. The proof is then completed.

\section{APPENDIX C \\ DeCREASING Monotonicity of $\theta_{L}$}

From (27), the optimal energy efficiency can be rewritten as

$$
\eta_{I, L}^{*}=\mathcal{K} \theta_{L} .
$$

where $\mathcal{K}=\psi \mathcal{T}_{0} \frac{\left(R^{*}-\mathcal{T}_{0}\right)^{c}}{R^{* c}\left(2^{R^{*}}-1\right)}$ and is independent of $\rho$. Accordingly, the monotonicity of $\theta_{L}$ with respect to $\rho$ is the same as that of $\eta_{I, L}^{*}$. With the same monotonicity, we can prove the decreasing monotonicity of $\theta_{L}$ through the analysis of the monotonicity of $\eta_{I, L}^{*}$ as follows.

Specifically, as proved in [30, Lemma 3], the asymptotic outage probability is an increasing function of $\rho$. Thus it follows from (8) that the energy efficiency $\eta_{I, L}$ is a decreasing function of $\rho$. To proceed with the proof, we assume two distinct time correlation coefficients $\rho_{1}$ and $\rho_{2}$ with $\rho_{1}>\rho_{2}$. Denote the optimal solution to the original problem (9) under the time correlation of $\rho_{1}$ and the corresponding optimal energy efficiency as $\left(P_{1}{ }^{*}, \cdots, P_{L}{ }^{*}, R^{*}\right)$ and $\eta_{I, L, \rho_{1}}^{*}=\eta_{I, L, \rho_{1}}\left(P_{1}^{*}, \cdots, P_{L}{ }^{*}, R^{*}\right)$, respectively. When the channel time correlation is reduced to $\rho_{2}$, the solution $\left(P_{1}{ }^{*}, \cdots, P_{L}{ }^{*}, R^{*}\right)$ is still feasible to the problem (9) since it satisfies all the constraints in (9). With the decreasing monotonicity of $\eta_{I, L}$ with respect to $\rho$, we have $\eta_{I, L, \rho_{1}}\left(P_{1}{ }^{*}, \cdots, P_{L}{ }^{*}, R^{*}\right)<\eta_{I, L, \rho_{2}}\left(P_{1}{ }^{*}, \cdots, P_{L}{ }^{*}, R^{*}\right)$. Moreover, when the time correlation of $\rho_{2}$ is considered, the optimization in (9) will definitely lead to the optimal energy efficiency $\eta_{I, L, \rho_{2}}^{*}$ not lower than $\eta_{I, L, \rho_{2}}\left(P_{1}{ }^{*}, \cdots, P_{L}{ }^{*}, R^{*}\right)$, i.e., $\eta_{I, L, \rho_{2}}^{*} \geq \eta_{I, L, \rho_{2}}\left(P_{1}{ }^{*}, \cdots, P_{L}{ }^{*}, R^{*}\right)$. Therefore, we have $\eta_{I, L, \rho_{2}}^{*}>\eta_{I, L, \rho_{1}}^{*}$. It means that the optimal energy efficiency $\eta_{I, L}^{*}$ is a monotonically decreasing function of the time correlation $\rho$. Combining this monotonicity with (77), we can conclude that $\theta_{L}$ is a decreasing functions of $\rho$ and the proof is finally completed.

\section{APPENDIX D \\ PROOF OF PROPERTY 1}

Consider two different maximal numbers of transmissions $L_{1}$ and $L_{2}$ with $L_{1} \leq L_{2}$. We denote the optimal transmission powers and rate and the corresponding optimal energy efficiency under the case with maximal $L_{1}$ transmissions as $\mathcal{S}_{1}^{*}=\left(P_{1}^{*}, \cdots, P_{L_{1}}^{*}, R^{*}\right)$ and $\eta_{L_{1}}^{*}$, respectively. Now when the maximal number of transmissions increases to $L_{2}$, we construct a solution of transmission powers and rate as $\mathcal{S}_{2}=\left(P_{1}^{*}, \cdots, P_{L_{1}}^{*}, P_{L_{1}+1}, \cdots, P_{L_{2}}, R^{*}\right)$ where $P_{L_{1}+1}=$ $\cdots=P_{L_{2}}=0$. Clearly from the definition of outage probability in (3), we have $p_{\text {out }, L_{1}}=\cdots=p_{\text {out }, L_{2}}$. When the maximal number of transmissions is $L_{2}, \mathcal{S}_{2}$ constitues a feasible point of the problem (9), since $\mathcal{S}_{2}$ satisfies both outage and goodput constraints. Denote its corresponding energy efficiency as $\eta_{L_{2}}\left(\mathcal{S}_{2}\right)$. We directly have $\eta_{L_{2}}\left(\mathcal{S}_{2}\right)=\eta_{L_{1}}^{*}$. 
Through the optimization in (9), when the maximal number of transmissions is $L_{2}$, we can definitily find the optimal energy efficiency $\eta_{L_{2}}^{*}$ not lower than $\eta_{L_{2}}\left(\mathcal{S}_{2}\right)$, i.e., $\eta_{L_{2}}^{*} \geq \eta_{L_{2}}\left(\mathcal{S}_{2}\right)$. It then follows $\eta_{L_{2}}^{*} \geq \eta_{L_{1}}^{*}$, which means that the optimal energy efficiency is non-decreasing function of the maximum number of transmissions and thus completes the proof.

\section{APPENDIX E \\ PROOF OF THEOREM 3}

\section{A. Proof of (28)}

Taking the limit $L \rightarrow \infty$ of both sides of (27) yields

$$
\begin{aligned}
\eta_{I, \infty}^{*} & =\lim _{L \rightarrow \infty} \psi \theta_{L} \mathcal{T}_{0} \frac{\left(R^{*}-\mathcal{T}_{0}\right)^{c}}{R^{* c}\left(2^{R^{*}}-1\right)} \\
& =\lim _{L \rightarrow \infty} \frac{\left(2^{\frac{L}{1-2^{-L}}-2}\right) \theta_{L} \mathcal{T}_{0}}{2^{L}-1} \lim _{L \rightarrow \infty} \frac{\left(R^{*}-\mathcal{T}_{0}\right)^{c}}{R^{*^{c}}\left(2^{R^{*}}-1\right)} \\
& =\frac{\theta_{\infty} \mathcal{T}_{0}}{4} \lim _{L \rightarrow \infty} \frac{\left(R^{*}-\mathcal{T}_{0}\right)^{c}}{R^{* c}\left(2^{R^{*}}-1\right)},
\end{aligned}
$$

where $\theta_{\infty} \triangleq \lim _{L \rightarrow \infty} \theta_{L}$ and the existence of $\theta_{\infty}$ will be proved in Appendix E-B. As $L \rightarrow \infty$, we surely have $\Delta=\min \left\{\varepsilon, 2^{-L}\right\}=2^{-L}$. Recalling $\mathcal{T}_{0}<R^{*} \leq \frac{\mathcal{T}_{0}}{1-\Delta}$, $\lim _{L \rightarrow \infty} R^{*}=\mathcal{T}_{0}$ follows by applying squeeze theorem. Plugging this into (78) along with $\lim _{L \rightarrow \infty} c=0$ leads to

$$
\eta_{I, \infty}^{*}=\frac{\theta_{\infty} \mathcal{T}_{0}}{4\left(2^{\mathcal{T}_{0}}-1\right)} \lim _{L \rightarrow \infty}\left(R^{*}-\mathcal{T}_{0}\right)^{c} .
$$

Noticing that both $R^{*}-\mathcal{T}_{0}$ and $c$ in (79) converge to zero, the occurrence of the limit $0^{0}$ complicates the derivations. Fortunately, based on Theorem 2, we have $R^{*}=\varphi^{-1}(0)$ when $L \rightarrow \infty$ as $\varepsilon$ becomes greater than $2^{-L}$. It implies that $R^{*}$ is a zero point of $\varphi(R)$, i.e., $\varphi\left(R^{*}\right)=0$. Following the definition of $\varphi(R)$, we then have

$$
R^{*}=\mathcal{T}_{0}+c \mathcal{T}_{0} \Xi\left(R^{*}\right),
$$

where $\Xi(R)=\frac{2^{R}-1}{\ln (2) R 2^{R}}$. Now putting (80) into (79) yields

$$
\begin{aligned}
\eta_{I, \infty}^{*} & =\frac{\theta_{\infty} \mathcal{T}_{0}}{4\left(2^{\mathcal{T}_{0}}-1\right)} \lim _{L \rightarrow \infty}\left(c \mathcal{T}_{0} \Xi\left(R^{*}\right)\right)^{c} \\
& =\frac{\theta_{\infty} \mathcal{T}_{0}}{4\left(2^{\mathcal{T}_{0}}-1\right)} \lim _{L \rightarrow \infty} c^{c}\left(\lim _{L \rightarrow \infty} \mathcal{T}_{0} \Xi\left(R^{*}\right)\right)^{\lim _{L \rightarrow \infty} c} \\
& =\frac{\theta_{\infty} \mathcal{T}_{0}}{4\left(2^{\mathcal{T}_{0}}-1\right)} .
\end{aligned}
$$

The result in (28) then directly follows by combining (81) with Property 1.

Clearly, $\eta_{I, \infty}^{*}$ is a decreasing function of the goodput threshold $\mathcal{T}_{0}$. Moreover, when $\sigma_{1}{ }^{2}=\cdots=\sigma_{L}{ }^{2}=1$ and $\rho=0$, we have $\theta_{L}=1$ based on its definition in (22). As shown in Appendix $\mathrm{C}, \theta_{L}$ is a decreasing function of the time correlation coefficient $\rho$. Therefore, when $\sigma_{1}{ }^{2}=\cdots=\sigma_{L}{ }^{2}=1$ with $L \rightarrow \infty, \theta_{\infty}$ would be generally lower than or equal to that for the case with $\rho=0$. That is, $\theta_{\infty} \leq 1$. Applying this inequality into the maximal energy efficiency (81) and using the decreasing monotonicity of $\eta_{I, \infty}^{*}$ with respect to $\mathcal{T}_{0}$, it is easy to get (29).

\section{B. Existence of $\theta_{\infty}$}

Based on the definition in (22), $\theta_{\infty}$ can be written as

$$
\theta_{\infty}=\lim _{L \rightarrow \infty} \underbrace{\prod_{k=1}^{L}\left(\sigma_{k}^{2}\right)^{2^{-k}}}_{\omega_{L}} \lim _{L \rightarrow \infty} \underbrace{\prod_{k=1}^{L}\left(\frac{\ell(k, \rho)}{\ell(k-1, \rho)}\right)^{2^{-k}}}_{\vartheta_{L}}
$$

Here $\omega_{L}$ can be rewritten as $\omega_{L}=\exp \left(\sum_{k=1}^{L} 2^{-k} \ln {\sigma_{k}}^{2}\right)$. Since the channel power gain $\sigma_{1}^{2}, \cdots, \sigma_{\infty}^{2}$ are generally bounded in practice and every absolutely convergent series is convergent [35], the exponent $\sum_{k=1}^{L} 2^{-k} \ln \sigma_{k}{ }^{2}$ absolutely converges as $L \rightarrow \infty$. It follows that $\omega_{L}$ converges as $L \rightarrow \infty$, i.e., the limit of $\omega_{L}$ for $L \rightarrow \infty$ exists.

With respect to the term $\vartheta_{L}$, following the definition of $\ell(k, \rho)$ in (6), we have

$$
\frac{\ell(k, \rho)}{\ell(k-1, \rho)}=\frac{1+\left(1-\rho^{2(k-1)}\right) \sum_{t=1}^{k-1} \frac{\rho^{2(t-1)}}{1-\rho^{2(t-1)}}}{1+\sum_{t=1}^{k-1} \frac{\rho^{2(t-1)}}{1-\rho^{2(t-1)}}} \leq 1 .
$$

Accordingly, the sequence $\left\{\vartheta_{L}\right\}$ decreases with $L$ and is lower bounded as $\vartheta_{L} \geq 0$. Therefore, the limit $\lim _{L \rightarrow \infty} \vartheta_{L}$ exists. The existence of both the limits $\lim _{L \rightarrow \infty} \vartheta_{L}$ and $\lim _{L \rightarrow \infty} \omega_{L}$ then guarantees the existence $\theta_{\infty}$ and completes the proof.

\section{APPENDIX F \\ DERIVATION OF $\kappa_{\infty}$}

Noticing $2^{-k}>0$ together with $\kappa_{L}=\prod_{k=1}^{L} k^{2^{-k}}, \kappa_{L}$ is readily found to be an increasing sequence, such that $\kappa_{1}<$ $\kappa_{2}<\cdots<\kappa_{\infty}$. To prove the convergence of the sequence $\kappa_{L}$, it suffices to prove that $\kappa_{\infty}$ is upper bounded. To this end, $\kappa_{\infty}$ is rewritten as $\kappa_{\infty}=e^{\sum_{k=1}^{\infty} 2^{-k} \ln (k)}$. Then applying Jensen's inequality yields

$$
\kappa_{\infty} \leq e^{\ln \left(\sum_{k=1}^{\infty} k 2^{-k}\right)}=\sum_{k=1}^{\infty} k 2^{-k} .
$$

By using [39, Eq.0.231], we have $\kappa_{\infty} \leq 2$. Accordingly, the sequence $\kappa_{L}$ is convergent, and the limit $\kappa_{\infty}=\lim _{L \rightarrow \infty} \kappa_{L}$ exists. It is difficult to evaluate $\kappa_{\infty}$ exactly, however $\kappa_{\infty}$ can be approximated as $\kappa_{L}$ with a large $L$. To guarantee the approximate accuracy, $L$ should be properly chosen to achieve a sufficiently low approximation error which is defined as

$$
e_{L} \triangleq \frac{\kappa_{\infty}-\kappa_{L}}{\kappa_{\infty}}=1-\prod_{k=L+1}^{\infty} k^{2^{-k}}
$$

Clearly, the approximation error $e_{L}$ decreases with $L$, and is upper bounded as

$$
\begin{aligned}
e_{L} & =1-e^{-2^{-L}} \sum_{k=L+1}^{\infty} \frac{2^{-k}}{2^{-L}} \ln (k) \\
& \leq 1-e^{-2^{-L} \ln \left(\sum_{k=L+1}^{\infty} \frac{2^{-k}}{2^{-L}} k\right)} \\
& =1-e^{-2^{-L} \ln \left(\sum_{k=1}^{\infty} 2^{-k} k+L \sum_{k=1}^{\infty} 2^{-k}\right)} \\
& =1-(L+2)^{-2^{-L}}
\end{aligned}
$$


wherein the inequality holds by using Jensen's inequality. Based on (86), by setting $L=20$, the approximation error is upper bounded as $e_{L} \leq 2.95 * 10^{-6}$. Thus $\kappa_{\infty}$ can be approximated as $\kappa_{\infty} \approx \kappa_{20}=1.6617$ with approximation error less than $2.95 * 10^{-6}$ which is sufficiently low for practical applications.

\section{APPENDIX G \\ Proof of Increasing Monotonicity of $\mathcal{G}_{k}(R)$}

To prove the increasing monotonicity of $\mathcal{G}_{k}(R)$, we resort to analyze its first order derivative of $\mathcal{G}_{k}(R)$ with respect to $R$, given by

$$
\mathcal{G}_{k}^{\prime}(R)=\frac{\mathcal{U}_{k}(R)}{\left(R g_{k-1}(R)\right)^{2}},
$$

where $\mathcal{U}_{k}(R)=R g_{k}{ }^{\prime}(R) g_{k-1}(R)-R g_{k-1}{ }^{\prime}(R) g_{k}(R)-$ $g_{k-1}(R) g_{k}(R)$ and $g_{k}{ }^{\prime}(R)$ can be further written by using Residue theorem as

$$
\begin{aligned}
g_{k}{ }^{\prime}(R) & =\frac{\ln (2)}{2 \pi \mathrm{i}} \int_{a-\mathrm{i} \infty}^{a+\mathrm{i} \infty} \frac{2^{R s}}{(s-1)^{k}} d s \\
& =\frac{\ln (2)(R \ln (2))^{k-1}}{(k-1) !} 2^{R}, k \geq 1 .
\end{aligned}
$$

Specifically when $k=1$, it follows from (7) that $\mathcal{U}_{k}(R)$ reduces to $\mathcal{U}_{1}(R)=R \ln (2) 2^{R}-2^{R}+1=$ $2^{R}\left(R \ln (2)-1+e^{-\ln (2) R}\right)$. Clearly by using the inequality $e^{-x} \geq 1-x$, we have $\mathcal{U}_{1}(R) \geq 0$. It means that $\mathcal{G}_{k}(R)$ is an increasing function when $k=1$. On the other hand, when $k \geq 2$, it becomes very difficult to determine the sign of $\mathcal{U}_{k}(R)$. To proceed, we turn to analyze the first order derivative of $\mathcal{U}_{k}(R)$ with respect to $R$ given as

$$
\begin{aligned}
\mathcal{U}_{k}^{\prime}(R)=R g_{k}{ }^{\prime \prime}(R) g_{k-1}(R) & \\
& -\left(2 g_{k-1}{ }^{\prime}(R)+R g_{k-1}{ }^{\prime \prime}(R)\right) g_{k}(R),
\end{aligned}
$$

where similarly to (88), the second order derivative of $g_{k}(R)$ can also be written by using Residue theorem as

$$
\begin{gathered}
g_{k}^{\prime \prime}(R)=\frac{(\ln 2)^{2}}{2 \pi \mathrm{i}} \int_{a-\mathrm{i} \infty}^{a+\mathrm{i} \infty} \frac{s 2^{R s}}{(s-1)^{k}} d s=\frac{(\ln 2)^{2}}{(k-1) !} \times \\
\left(2^{R}(R \ln 2)^{k-1}+(k-1) 2^{R}(R \ln 2)^{k-2}\right), k \geq 2 .
\end{gathered}
$$

Substituting (88) and (90) into (89), it follows that

$$
\begin{aligned}
\mathcal{U}_{k}^{\prime}(R) & =\frac{\ln (2)(R \ln 2)^{k-2} 2^{R}}{(k-2) !} \\
& \times \underbrace{\left(\begin{array}{l}
\frac{R \ln (2)}{(k-1)}(R \ln (2)+(k-1)) g_{k-1}(R) \\
-(R \ln (2)+k) g_{k}(R)
\end{array}\right)}_{\mathcal{J}(R)} .
\end{aligned}
$$

It can be proved that $\mathcal{J}(R)$ is an increasing and convex function, since its first and second order derivatives of $\mathcal{J}(R)$ with respect to $R$ satisfy

$$
\begin{aligned}
\mathcal{J}^{\prime}(R)= & \left(\frac{2 R(\ln (2))^{2}}{(k-1)}+\ln (2)\right) g_{k-1}(R) \\
& -\ln (2) g_{k}(R)-\frac{\ln (2)(R \ln (2))^{k-1}}{(k-1) !} 2^{R}
\end{aligned}
$$

$$
\mathcal{J}^{\prime \prime}(R)=\frac{2(\ln (2))^{2}}{(k-1)} g_{k-1}(R) \geq 0 .
$$

Noticing that $\mathcal{J}^{\prime \prime}(R) \geq 0$ and $g_{k}(0)=0$, thus $\mathcal{J}^{\prime}(R) \geq$ $\mathcal{J}^{\prime}(0)=0$ for $k \geq 2$. Analogously, we can sequentially prove $\mathcal{J}(R) \geq \mathcal{J}(0)=0, \mathcal{U}_{k}^{\prime}(R) \geq \mathcal{U}_{k}^{\prime}(0)=0, \mathcal{U}_{k}(R) \geq \mathcal{U}_{k}(0)=$ 0 , then we have $\mathcal{G}_{k}^{\prime}(R) \geq 0$. Thus the increasing monotonicity of $\mathcal{G}_{k}(R)$ follows as well for $k \geq 2$. The proof is eventually completed.

\section{APPENDIX H \\ PROOF OF PROPERTY 2}

Prior to proving (38), the following upper and lower bounds associated with $\frac{g_{k}(R)}{g_{k-1}(R)}$ are obtained first.

A. $\frac{g_{k}(R)}{g_{k-1}(R)} \leq \frac{R \ln (2)}{k-1}$

To prove this inequality, it suffices to show that $\mathfrak{A}(R) \triangleq$ $\frac{R \ln (2)}{k-1} g_{k-1}(R)-g_{k}(R) \geq 0$. By taking the first order derivative of $\mathfrak{A}(R)$ with respect to $R$, it follows that

$$
\begin{aligned}
\mathfrak{A}^{\prime}(R) & =\frac{\ln (2)}{k-1} g_{k-1}(R)+\frac{R \ln (2)}{k-1} g_{k-1}{ }^{\prime}(R)-g_{k}{ }^{\prime}(R) \\
& =\frac{\ln (2)}{k-1} g_{k-1}(R) \geq 0 .
\end{aligned}
$$

where the second equality holds by using (88). Accordingly, $\mathfrak{A}(R)$ is an increasing function, which implies $\mathfrak{A}(R) \geq$ $\mathfrak{A}(0)=0$. Then the upper bound of $\frac{g_{k}(R)}{g_{k-1}(R)}$ holds.

B. $\frac{g_{k}(R)}{g_{k-1}(R)} \geq \frac{R \ln (2)}{k}$

To prove this lower bound, it is equivalent to prove $\mathfrak{B}(R) \triangleq$ $\frac{R \ln (2)}{k} g_{k-1}(R)-g_{k}(R) \leq 0$. To this end, taking the first order derivative of $\mathfrak{B}(R)$ with respect to $R$, we have

$$
\mathfrak{B}^{\prime}(R)=\frac{\ln (2)}{k} g_{k-1}(R)-\frac{\ln (2)(R \ln (2))^{k-1} 2^{R}}{k !} .
$$

Then taking the second order derivative of $\mathfrak{B}(R)$ with respect to $R$ yields

$$
\mathfrak{B}^{\prime \prime}(R)=-\frac{(\ln (2))^{2}(R \ln (2))^{k-1} 2^{R}}{k !} \leq 0
$$

Combining (95) and (96), it is clearly found that $\mathfrak{B}(R)$ is a decreasing function, which indicates $\mathfrak{B}(R) \leq \mathfrak{B}(0)=0$. Thus the lower bound of $\frac{g_{k}(R)}{g_{k-1}(R)}$ is proved.

As a consequence, substituting the upper and lower bounds of $\frac{g_{k}(R)}{g_{k-1}(R)}$ into $\Lambda(R)$ directly yields the upper and lower bounds of $\Lambda(R)$, respectively, as shown in (38).

\section{APPENDIX I \\ ProOf OF (44)}

For the proof, it suffices to show that $\mathfrak{C}(R) \triangleq g_{k}(R)-$ $\frac{2^{R}-1}{k} g_{k-1}(R) \leq 0$. Taking the first order derivative of $\mathfrak{C}(R)$ with respect to $R$ gives

$$
\begin{aligned}
\mathfrak{C}^{\prime}(R) & =2^{R} \ln (2)\left(\frac{(R \ln (2))^{k-1}}{(k-1) !}-\right. \\
& \left.\frac{k-1}{k} \frac{\left(2^{R}-1\right)(R \ln (2))^{k-2}}{(k-1) !}-\frac{g_{k-1}(R)}{k}\right) .
\end{aligned}
$$


By using the lower bound of $\frac{g_{k}(R)}{g_{k-1}(R)}$ in Appendix H-B, namely $\frac{g_{k}(R)}{g_{k-1}(R)} \geq \frac{R \ln (2)}{k}$, it follows that

$$
\begin{aligned}
& g_{k-1}(R) \geq \frac{R \ln (2)}{k-1} g_{k-2}(R) \geq \cdots \\
& \geq \frac{(R \ln (2))^{k-2}}{(k-1) !} g_{1}(R)=\frac{(R \ln (2))^{k-2}\left(2^{R}-1\right)}{(k-1) !} .
\end{aligned}
$$

Substituting (98) into (97) yields

$$
\mathfrak{C}^{\prime}(R) \leq \frac{2^{R} \ln (2)(R \ln (2))^{k-2}}{(k-1) !}\left(R \ln (2)-\left(2^{R}-1\right)\right) \leq 0 .
$$

Thus $\mathfrak{C}(R)$ turns out to be a decreasing function of $R$, which implies $\mathfrak{C}(R) \leq \mathfrak{C}(0)=0$. The proof is then completed.

\section{REFERENCES}

[1] R. Sassioui, M. Jabi, L. Szczecinski, L. B. Le, M. Benjillali, and B. Pelletier, "HARQ and AMC: Friends or foes?" IEEE Trans. Commun., vol. 65, no. 2, pp. 635-650, Feb. 2017.

[2] Y. Li, M. C. Gursoy, and S. Velipasalar, "On the throughput of hybridARQ under statistical queuing constraints," IEEE Trans. Veh. Technol., vol. 64 , no. 6 , pp. $2725-2732$, Jun. 2015.

[3] J. Harsini, F. Lahouti, M. Levorato, and M. Zorzi, "Analysis of noncooperative and cooperative type II hybrid ARQ protocols with AM$\mathrm{C}$ over correlated fading channels," IEEE Trans. Wireless Commun., vol. 10, no. 3, pp. 877-889, Mar. 2011.

[4] J. Choi and J. Ha, "On the energy efficiency of AMC and HARQ-IR with QoS constraints," IEEE Trans. Veh. Technol., vol. 62, no. 7, pp. 3261-3270, Sep. 2013.

[5] G. Caire and D. Tuninetti, "The throughput of hybrid-ARQ protocols for the Gaussian collision channel," IEEE Trans. Inf. Theory, vol. 47, no. 5, pp. 1971-1988, Jul. 2001.

[6] S. H. Kim, S. J. Lee, and D. K. Sung, "Rate-adaptation-based cooperative hybrid-ARQ relaying scheme in Rayleigh block-fading channels," IEEE Trans. Veh. Technol., vol. 60, no. 9, pp. 4640-4645, Nov. 2011.

[7] _ , "Low-complexity rate selection of HARQ with chase combining in Rayleigh block-fading channels," IEEE Trans. Veh. Technol., vol. 62, no. 6, pp. 2818-2824, Jul. 2013.

[8] P. Wu and N. Jindal, "Performance of hybrid-ARQ in block-fading channels: a fixed outage probability analysis," IEEE Trans. Commun., vol. 58, no. 4, pp. 1129-1141, Apr. 2010.

[9] A. Chelli and M. Alouini, "On the performance of hybrid-ARQ with incremental redundancy and with code combining over relay channels," IEEE Trans. Wireless Commun., vol. 12, no. 8, pp. 3860-3871, Aug. 2013.

[10] P. Larsson, L. K. Rasmussen, and M. Skoglund, "Throughput analysis of ARQ schemes in Gaussian block fading channels," IEEE Trans. Commun., vol. 62, no. 7, pp. 2569-2588, Jul. 2014.

[11] _ _ "Throughput analysis of hybrid-ARQ — a matrix exponential distribution approach," IEEE Trans. Commun., vol. 64, no. 1, pp. 416-428, Jan. 2016.

[12] L. Szczecinski, S. R. Khosravirad, P. Duhamel, and M. Rahman, "Rate allocation and adaptation for incremental redundancy truncated HARQ," IEEE Trans. Commun., vol. 61, no. 6, pp. 2580-2590, Jun. 2013.

[13] J. Choi, D. To, Y. Wu, and S. Xu, "Energy-delay tradeoff for wireless relay systems using HARQ with incremental redundancy," IEEE Trans. Wireless Commun., vol. 12, no. 2, pp. 561-573, Feb. 2013.

[14] A. Chelli, E. Zedini, M.-S. Alouini, J. Barry, and M. Patzold, "Performance and delay analysis of hybrid ARQ with incremental redundancy over double Rayleigh fading channels," IEEE Trans. Wireless Commun., vol. 13, no. 11, pp. 6245-6258, Nov. 2014.

[15] G. Y. Li, Z. Xu, C. Xiong, C. Yang, S. Zhang, Y. Chen, and S. Xu, "Energy-efficient wireless communications: tutorial, survey, and open issues," IEEE Wireless Commun., vol. 18, no. 6, pp. 28-35, Dec. 2011.

[16] J. Wu, G. Wang, and Y. R. Zheng, "Energy efficiency and spectral efficiency tradeoff in type-I ARQ systems," IEEE J. Sel. Areas Commun., vol. 32, no. 2, pp. 356-366, Feb. 2014.

[17] J. Choi, "Energy-delay tradeoff comparison of transmission schemes with limited CSI feedback," IEEE Trans. Wireless Commun., vol. 12, no. 4, pp. 1762-1773, Apr. 2013.
[18] M. Jabi, M. Benjillali, L. Szczecinski, and F. Labeau, "Energy efficiency of adaptive HARQ," IEEE Trans. Commun., vol. 64, no. 2, pp. 818-831, Feb. 2016.

[19] Z. Hu, C. Yuan, F. Zhu, and F. Gao, "Weighted sum transmit power minimization for full-duplex system with SWIPT and self-energy recycling," IEEE Access, vol. 4, pp. 4874-4881, Sep. 2016.

[20] A. Basit, G. A. S. Sidhu, A. Mahmood, and F. Gao, "Efficient and autonomous energy management techniques for the future smart homes," IEEE Trans. Smart Grid, vol. 8, no. 2, pp. 917-926, Mar. 2017.

[21] X. Ge, J. Yang, H. Gharavi, and Y. Sun, "Energy efficiency challenges of 5G small cell networks," IEEE Commun. Mag., vol. 55, no. 5, pp. 184-191, May 2017.

[22] R. Zi, X. Ge, J. Thompson, C.-X. Wang, H. Wang, and T. Han, "Energy efficiency optimization of $5 \mathrm{G}$ radio frequency chain systems," IEEE $J$. Sel. Areas Commun., vol. 34, no. 4, pp. 758-771, Apr. 2016.

[23] X. Ge, R. Zi, H. Wang, J. Zhang, and M. Jo, "Multi-user massive MIMO communication systems based on irregular antenna arrays," IEEE Trans. Wireless Commun., vol. 15, no. 8, pp. 5287-5301, Aug. 2016.

[24] S. M. Kim, W. Choi, T. W. Ban, and D. K. Sung, "Optimal rate adaptation for hybrid ARQ in time-correlated Rayleigh fading channels," IEEE Trans. Wireless Commun., vol. 10, no. 3, pp. 968-979, Mar. 2011.

[25] H. Jin, C. Cho, N.-O. Song, and D. K. Sung, "Optimal rate selection for persistent scheduling with HARQ in time-correlated Nakagami-m fading channels," IEEE Trans. Wireless Commun., vol. 10, no. 2, pp. 637-647, Feb. 2011.

[26] Z. Shi, H. Ding, S. Ma, and K.-W. Tam, "Analysis of HARQ-IR over time-correlated Rayleigh fading channels," IEEE Trans. Wireless Commun., vol. 14, no. 12, pp. 7096-7109, Dec. 2015.

[27] Z. Shi, H. Ding, S. Ma, K.-W. Tam, and S. Pan, "Inverse moment matching based analysis of cooperative HARQ-IR over time-correlated Nakagami fading channels," IEEE Trans. Veh. Technol., vol. 66, no. 5, pp. 3812-3828, May 2017.

[28] Z. Shi, S. Ma, and K.-W. Tam, "Outage analysis on type I HARQ over time-correlated Rayleigh fading channels," in Proc. IEEE/CIC International Conference on Communications in China (ICCC'15), Nov. 2015, pp. 1-6.

[29] Z. Shi, S. Ma, F. Hou, K.-W. Tam, and Y.-C. Wu, "Optimal power allocation for HARQ schemes over time-correlated Nakagami-m fading channels," in Proc. IEEE International Conference on Communication Systems (ICCS'16), Dec. 2016, pp. 1-6.

[30] Z. Shi, S. Ma, G. Yang, K. W. Tam, and M. Xia, "Asymptotic outage analysis of HARQ-IR over time-correlated Nakagami-m fading channels," IEEE Trans. Wireless Commun., vol. 16, no. 9, pp. 6119-6134, Sep. 2017.

[31] M. Zorzi and R. R. Rao, "On the use of renewal theory in the analysis of ARQ protocols," IEEE Trans. Commun., vol. 44, no. 9, pp. 1077-1081, Sep. 1996.

[32] W. Rui and V. K. Lau, "Combined cross-layer design and HARQ for multiuser systems with outdated channel state information at transmitter (CSIT) in slow fading channels," IEEE Trans. Wireless Commun., vol. 7, no. 7, pp. 2771-2777, Jul. 2008.

[33] H. Zheng and H. Viswanathan, "Optimizing the ARQ performance in downlink packet data systems with scheduling," IEEE Trans. Wireless Commun., vol. 4, no. 2, pp. 495-506, Mar. 2005.

[34] D. P. Palomar and M. Chiang, "A tutorial on decomposition methods for network utility maximization," IEEE J. Sel. Areas Commun., vol. 24, no. 8, pp. 1439-1451, Aug. 2006.

[35] W. Rudin et al., Principles of mathematical analysis. McGraw-Hill New York, 1964, vol. 3.

[36] R. Tajan, "Hybrid-ARQ mechanisms in a radio-cognitive context." Ph.D. dissertation, Université de Cergy Pontoise, 2013.

[37] D. Tuninetti, "On the benefits of partial channel state information for repetition protocols in block fading channels," IEEE Trans. Inf. Theory, vol. 57, no. 8, pp. 5036-5053, Aug. 2011.

[38] A. Goldsmith, Wireless communications. Cambridge university press, 2005.

[39] I. S. Gradshteyn, I. M. Ryzhik, A. Jeffrey, D. Zwillinger, and S. Technica, Table of integrals, series, and products. Academic press New York, 1965, vol. 6 . 